\title{
A comparison between triplet and doublet chemotherapy in improving the survival of patients with advanced gastric cancer: a systematic review and meta-analysis
}

Xinjian Guo', Fuxing Zhao', Xinfu Ma', Guoshuang Shen', Dengfeng Ren ${ }^{1}$, Fangchao Zheng ${ }^{1,2,3}$, Feng Du ${ }^{4}$, Ziyi Wang ${ }^{1}$, Raees Ahmad ${ }^{1}$, Xinyue Yuan ${ }^{1}$, Junhui Zhao ${ }^{1 *}$ and Jiuda Zhao ${ }^{1 *}$

\begin{abstract}
Background: Chemotherapy can improve the survival of patients with advanced gastric cancer. However, whether triplet chemotherapy can further improve the survival of patients with advanced gastric cancer compared with doublet chemotherapy remains controversial. This study reviewed and updated all published and eligible randomized controlled trials (RCTs) to compare the efficacy, prognosis, and toxicity of triplet chemotherapy with doublet chemotherapy in patients with advanced gastric cancer.

Methods: RCTs on first-line chemotherapy in advanced gastric cancer on PubMed, Embase, and the Cochrane Register of Controlled Trials and all abstracts from the annual meetings of the European Society for Medical Oncology (ESMO) and the American Society of Clinical Oncology conferences up to October 2018 were searched. The primary outcome was overall survival, while the secondary outcomes were progression-free survival (PFS), time to progress (TTP), objective response rate (ORR), and toxicity.

Results: Our analysis included 23 RCTs involving 4540 patients and 8 types of triplet and doublet chemotherapy regimens, and systematic review and meta-analysis revealed that triplet chemotherapy was superior compared with doublet chemotherapy in terms of improving median OS ( $H R=0.92 ; 95 \% \mathrm{Cl}, 0.86-0.98 ; P=0.02)$ and PFS ( $H R=0.82$; 95\% Cl, 0.69-0.97; $P=0.02)$ and TTP $(H R=0.92 ; 95 \% C l, 0.86-0.98 ; P=0.02)$ and $\mathrm{ORR}(\mathrm{OR}=1.21 ; 95 \% \mathrm{Cl}, 1.12-1.31$; $P<0.0001)$ among overall populations. Compared with doublet chemotherapy, subgroup analysis indicated that OS improved with fluoropyrimidine-based $(H R=0.80 ; 95 \% \mathrm{Cl}, 0.66-0.96 ; P=0.02)$, platinum-based $(\mathrm{HR}=0.75 ; 95 \% \mathrm{Cl}$, 0.57-0.99; $P=0.04)$, and other drug-based triplet $(\mathrm{HR}=0.79 ; 95 \% \mathrm{Cl}, 0.69-0.90 ; P=0.0006)$ chemotherapies while not with anthracycline-based $(H R=0.70 ; 95 \% \mathrm{Cl}, 0.42-1.15 ; P=0.16)$, mitomycin-based $(\mathrm{HR}=0.81 ; 95 \% \mathrm{Cl}, 0.47-1.39 ; P=$ $0.44)$, taxane-based $(H R=0.91 ; 95 \% \mathrm{Cl}, 0.81-1.01 ; P=0.07)$, and irinotecan-based triplet $(\mathrm{HR}=1.01 ; 95 \% \mathrm{Cl}, 0.82-1.24$; $P=0.94)$ chemotherapies. For different patients, compared with doublet chemotherapy, triplet chemotherapy improved OS ( $\mathrm{HR}=0.89 ; 95 \% \mathrm{Cl}, 0.81-0.99 ; P=0.03)$ among Western patients but did not improve $(\mathrm{HR}=0.96 ; 95 \%$ $\mathrm{Cl}, 0.86-1.07 ; P=0.47)$ that among Asian patients.
\end{abstract}

Conclusions: Compared with doublet chemotherapy, triplet chemotherapy improved OS, PFS, TTP, and ORR in patients with advanced gastric cancer in the population overall, and improved OS in Western but not in Asian patients.

Keywords: Advanced gastric cancer, Triplet chemotherapy, Doublet chemotherapy, Meta-analysis, First-line chemotherapy

\footnotetext{
*Correspondence: zhao699@126.com; jiudazhao@126.com

${ }^{1}$ Affiliated Hospital of Qinghai University, Affiliated Cancer Hospital of

Qinghai University, Xining 810000, China

Full list of author information is available at the end of the article
}

(c) The Author(s). 2019 Open Access This article is distributed under the terms of the Creative Commons Attribution 4.0 International License (http://creativecommons.org/licenses/by/4.0/), which permits unrestricted use, distribution, and reproduction in any medium, provided you give appropriate credit to the original author(s) and the source, provide a link to the Creative Commons license, and indicate if changes were made. The Creative Commons Public Domain Dedication waiver (http://creativecommons.org/publicdomain/zero/1.0/) applies to the data made available in this article, unless otherwise stated. 


\section{Background}

Gastric cancer is a significant health burden worldwide. Global Cancer Statistics 2018 estimates that there will be $1,033,701$ (5.7\% of all sites) new cases and 782,685 (8.2 of all sites) deaths due to gastric cancer in 2018 [1]. Generally, 80-90\% of patients with gastric cancer are diagnosed at an advanced stage, implying that the tumor either cannot be resected through operation or developed a recurrence or metastasis after surgery $[2,3]$. The prognosis of these patients remains very poor, and the median survival time is only about 12 months [3]. Several targeted therapies, such as the human epidermal growth factor receptor 2 (HER2) antibody trastuzumab and the anti-vascular endothelial growth factor receptor 2 drugs including ramucirumab and apatinib, and immunotherapies including pembrolizumab and nivolumab have shown efficacy in metastatic gastric cancer $[4,5]$. Though molecularly targeted treatment is promising for improving the survival of patients with advanced gastric cancer, the number of patients who appropriately receive this treatment is less considering the high heterogeneity and lack of targets in gastric cancer. Therefore, systemic chemotherapy remains the current main treatment in patients with advanced gastric cancer [6]. Especially for first-line setting, only trastuzumab or ramucirumab combined with chemotherapy is approved, with only about $10 \%$ of patients experiencing HER2 overexpression [7].

Chemotherapy can improve the survival of patients with advanced gastric cancer. Compared with best supportive care, systemic chemotherapy improves not only the survival but also the quality of life of the patients [2, 8]. According to the number of chemotherapeutic drugs included in the treatment method, chemotherapy regimens of patients with advanced gastric cancer are usually divided into singlet, doublet, and triplet chemotherapy. Combination chemotherapy has substantially higher objective response and survival rates than monotherapy [2, 8]. However, whether triplet chemotherapy can improve the survival of patients with advanced gastric cancer compared with doublet chemotherapy remains controversial considering the discrepancy among studies $[2,4,8]$. To date, nearly 30 studies have focused on this issue. Meta-analyses also show inconsistent results. For instance, one meta-analysis concludes that taxane-based triplet chemotherapy improves the survival of patients with advanced gastric cancer than doublet chemotherapy, while another meta-analysis does not support this $[8,9]$.

Several major international guidelines for advanced gastric cancer also have different recommendations concerning triplet or doublet chemotherapy. The European Society for Medical Oncology (ESMO) guidelines of 2016 state that both doublet and triplet chemotherapies belong to level I and grade A corresponding to levels of evidence and grades of recommendation, respectively, in patients with advanced gastric cancer [10]. However, the National Comprehensive Cancer Network guidelines (version 2.2018) suggest that doublet regimens are preferred and triplet regimens should be reserved for medically fit patients with good performance status (PS) [4]. Additionally, the Japanese gastric cancer treatment guidelines 2014 (version 4) only classifies triplet regimen as category 3 , implying that cannot be used in general practice [5]. The Chinese Society of Clinical Oncology guidelines for the diagnosis and treatment of primary gastric cancer (2018 edition) also suggest that triplet chemotherapy is an "optional strategy" but not a "basic strategy" [11]. With all of these uncertainties regarding the role of triplet regimen, as evidenced by the different guidelines discussed above, there is an urgent appeal of a new study on the definite role of triplet regimen in advanced gastric cancer. Such studies are still ongoing and have been published [12-14]. Nevertheless, two recent large-scale studies convey contrasting results. Wang et al. reported that modified DCF (docetaxel and cisplatin plus fluorouracil) regimen improved progressionfree survival (PFS) and overall survival (OS) in patients with treatment-naive advanced gastric cancer compared with cisplatin plus fluorouracil regimen [14]. Yasuhide Yamada et al. concluded that another modified DCF regimen (docetaxel and cisplatin plus S1) did not improve the OS of patients with untreated advanced gastric cancer compared with cisplatin plus S1 regimen [12].

Hence, whether triplet or doublet chemotherapy improves the survival of patients with advanced gastric cancer is still questionable in a first-line setting. Therefore, we conducted a systematic review and updated the meta-analysis of all published eligible randomized controlled trials (RCTs) to compare the efficacy, prognosis, and toxicity of triplet with doublet chemotherapy in patients with advanced gastric cancer.

\section{Methods \\ Study protocol}

The protocol of this systematic review has been registered on PROSPERO in September 2018 (registration, CRD42018110550).

\section{Literature search}

We searched PubMed, Embase, and the Cochrane Register of Controlled Trials (CENTRAL) up to October 2018. Studies were selected using the following search terms: "gastric or esophagogastric or gastroesophageal or gastroesophagus or stomach," "cancer or neoplasm or carcinoma or malignancy," "chemotherapy or chemotherapeutic or antineoplastic agent or antineoplastic drug," "randomized or randomised trial or randomized, controlled trial," and free text searches. No language limits were applied. Results were limited to RCTs that compared OS, PFS, 
objective response rate (ORR), and safety between triplet and doublet chemotherapy in patients with advanced gastric cancer. Additionally, all abstracts from the annual meetings of the ESMO and the American Society of Clinical Oncology (ASCO) conferences up to October 2018 were also searched. The eligible reports were independently identified by two reviewers (XFM and FXZ), and disagreements were discussed with a third reviewer (DFR) until consensus was reached. This systematic review was conducted according to the Preferred Reporting Items for Systematic Reviews and Meta-Analyses (PRISMA) statement [15-17].

\section{Study selection}

Studies meeting the following criteria of eligibility were included: 1) studies utilizing prospective phase II or III RCTs; 2) studies whose patients have pathologically proven advanced, recurrent, metastatic, or unresectable adenocarcinoma of the stomach or gastroesophageal junction; 3) studies with first-line chemotherapy setting; and 4) studies that compared at least two arms that consisted of the following chemotherapeutic drugs: fluoropyrimidine (F, either 5-fluorouracil [5-FU], capecitabine [Cap], or S-1), platinum (cisplatin [Cis] and oxaliplatin $[\mathrm{Ox}])$, taxane ([T] and paclitaxel), anthracycline (doxorubicin $[D]$ and epirubicin $[E])$, irinotecan (I), etoposide (E), semustine (Me), mitomycin (MMC), methotrexate (Mtx), uracil (U), or tegafur (Te). Studies that are retrospective or included patients receiving targeted treatment were excluded.

\section{Data extraction and quality assessment}

The primary outcome was OS, defined as the time from the date of random assignment to the date of death or last date of follow-up. Secondary outcomes were PFS; time to progress (TTP), defined as the duration from the date of random assignment to the date of events occurring; ORR, which estimates the rate of complete response plus partial response; and grade 3 to 4 adverse events (AEs). Treatment-related AEs defined the highest grade of toxicity per patient. AEs data, when available, were recorded if scored as grade 3-4 toxicity.

The methodological quality of all eligible studies was assessed using the Cochrane Risk of Bias Tool (version 5.1.0) [18, 19].

\section{Statistical analysis}

Survival analyses were conducted using the intention-totreat (ITT) population. A fixed effects model was used to calculate the pooled hazard ratio (HR) estimate. HRs for progression and death were combined using an inversevariance method based on a logarithmic conversion; 95\% confidence intervals (95\% CIs) were used to determine the standard error (SE), using the following formula: $\mathrm{SE}=95 \%$
$\mathrm{CI} / 1.96$. Statistical heterogeneity was tested with the Cochran Q test and quantified by the $I^{2}$ index. Heterogeneity was considered statistically significant when $P$ is less than 0.05 or $I^{2}$ is greater than $50 \%$. A random effects model was carried among trials with significant heterogeneity; otherwise, a fixed effects model was used. Publication bias was tested using funnel plots. When comparing triplet versus doublet chemotherapy, subgroup analyses including whether the regimens included fluorouracil (FU), platinum, anthracycline, taxane, irinotecan (I), MMC, and others and whether the studies included either Asian or Western patients were prespecified in advance in the registered protocol. Furthermore, the subgroup analysis comparing different chemotherapy combinations only included those triplet regimens having two generic drugs available in doublet regimens and investigated the effectiveness of irinotecan-based chemotherapy regimen in improving the survival of patients with gastric cancer considering the rarity of irinotecanbased study. RevMan v5.3 software was used to report all outcomes. All tests were performed two-sided, with a $P$ value less than 0.05 considered statistically significant.

\section{Results}

\section{Literature search and study characteristics}

A total of 9865 unique references were identified through searching PubMed, Embase, and the CENTRAL. After the exclusion of duplicate publications, 2231 unique references remained for further evaluation. Of these papers, 2207 were excluded because of the following reasons: these papers were solely reviews, RCTs were not available for these papers, and these papers did not compare doublet versus triplet regimen. The full texts of the remaining 24 articles were assessed. Ultimately, 23 articles involving 4540 patients with advanced gastric cancer were included in our systematic review [12, 14, 20-40]. A flowchart of study selection is shown in Fig. 1.

Table 1 shows the characteristics of the studies included in this meta-analysis. Generally, 23 studies were included. The total number of included patients in every study ranged from 25 to 741 . All RCTs satisfied the inclusion criteria and compared triplet combination versus doublet combination chemotherapy. Of the 23 included trials, two contained three groups, two triplet groups and one doublet group [24, 25]; one contained three groups, one triplet group and two doublet groups [27]; one contained four groups, two triplet groups and two doublet groups [29]; and the other were all two groups, one triplet group and one doublet group [12, 14, 20-23, 26, 28, 30-40].

Of these studies, 2380 were assigned to the triplet and 2160 to the doublet group. Median age was 51 to 70 years. In these studies, 2039 and 2501 (44.9 and 55.1\%, respectively) patients were Asians and Westerners, respectively. PS was well balanced in all studies. All patients had an ECOG PS of 0 or 1 . 


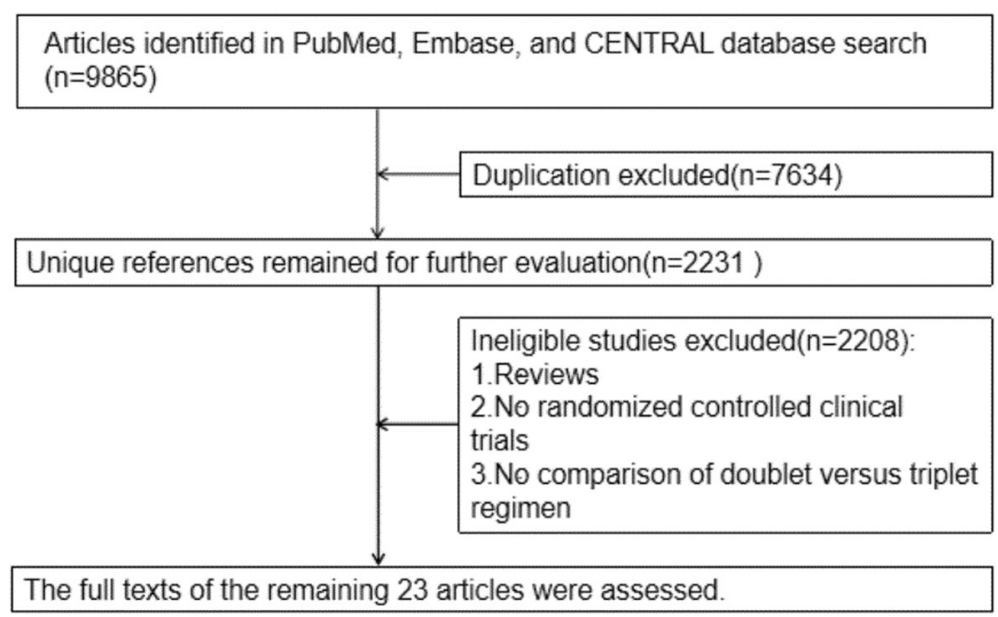

Fig. 1 A flowchart of study selection

Overall survival, progression-free survival, time to progress, and objective response rate

Twenty of the 23 trials reported OS in the study patients. OS was compared in 2126 patients treated wo received triplet chemotherapy with 1999 patients who received doublet chemotherapy. A significant reduction in the risk of death ( $\mathrm{HR}=0.92 ; 95 \% \mathrm{CI}, 0.86-0.98 ; P=0.02)$ was observed with triplet chemotherapy, as shown in Fig. 2 . Heterogeneity in the data was not observed $\left(P=0.08, \mathrm{I}^{2}=\right.$ $33 \%)$, which was assessed using a fixed effects model.

Ten of the 23 trials reported PFS in the study patients. The meta-analysis results showed that triplet chemotherapy also significantly improved PFS compared with doublet chemotherapy in patients $(\mathrm{HR}=0.82 ; 95 \% \mathrm{CI}$, 0.69-0.97; $P=0.02$, Fig. 3). Comparison was performed under the random effects model, because obvious heterogeneity was observed $\left(P<0.0001, \mathrm{I}^{2}=83 \%\right)$.

Five out of the 23 trials provided data regarding the TTP, while only one had HR. A meta-analysis was performed using fixed effects model to pool the HRs as there was no heterogeneity among trials $\left(P=0.39, \mathrm{I}^{2}=2 \%\right)$. The combined HR for TTP showed that triplet chemotherapy was superior compared with doublet combination regimen $(\mathrm{HR}=0.82 ; 95 \% \mathrm{CI}, 0.70-0.95 ; P=0.01$, Fig. 4).

All the 23 studies demonstrated ORR. The metaanalysis showed a significant improvement for ORR in triplet chemotherapy compared with doublet chemotherapy group $(\mathrm{OR}=1.21 ; 95 \% \mathrm{CI}, 1.12-1.31 ; P<0.0001$, Fig. 5). The $\mathrm{I}^{2}$ value of the heterogeneity test was $46 \%$, and a fixed effects model was used.

\section{Subgroup analysis}

We conducted a subgroup analysis according to the comparison of different triplet chemotherapy regimens containing two identical drugs with doublet regimens. Moreover, we also performed a subgroup analysis in patients who were from Asia or the Western. We summarized the results of our subgroup analysis for OS, PFS, and ORR in Additional file 1: Figure S1, Additional file 2: Figure S2 and Additional file 3: Figure S3 (Data not shown).

\section{Fluoropyrimidine-based triplet versus non- fluoropyrimidine-based doublet chemotherapy}

Four trials reported four fluoropyrimidine-based triplet chemotherapy compared with doublet chemotherapy $[20,24,25,29]$. The results of the subgroup analysis revealed that the addition of fluoropyrimidine in triplet chemotherapy regimens improved OS significantly but not PFS compared with the doublet chemotherapy $\left(\mathrm{HR}=0.80 ; 95 \% \mathrm{CI}, 0.66-0.96 ; P=0.02 ; \mathrm{I}^{2}=63 \%\right.$ vs. $\mathrm{HR}=0.56 ; 95 \% \mathrm{CI}, 0.21-1.46 ; P=0.24 ; \mathrm{I}^{2}=94 \%$, respectively, Additional file 1: Figure S1, Additional file 2: Figure S2). Additionally, fluoropyrimidine-based triplet regimens had a higher ORR than doublet chemotherapy $\left(\mathrm{OR}=1.60 ; 95 \% \mathrm{CI}, 1.23-2.09 ; P=0.0005 ; \mathrm{I}^{2}=0 \%\right.$, Additional file 3: Figure S3).

\section{Platinum-based triplet versus non-platinum-based doublet chemotherapy}

Among the included trials, three trials reported three platinum-based triplet chemotherapy compared with doublet chemotherapy $[23,36,40]$. The results of the subgroup analysis revealed that the addition of a platinum in triplet chemotherapy regimens had a significant improvement on OS compared with the doublet chemotherapy regimens $(\mathrm{HR}=0.75 ; 95 \% \mathrm{CI}, 0.57-0.99 ; P=$ $0.04 ; \mathrm{I}^{2}=0 \%$, Additional file 1: Figure S1). Moreover, platinum-based triplet chemotherapy was not superior in terms of ORR compared with doublet chemotherapy $\left(\mathrm{OR}=1.39 ; 95 \% \mathrm{CI}, 0.98-1.97 ; P=0.06 ; \mathrm{I}^{2}=54 \%\right.$, Additional file 3: Figure S3). 
Table 1 Characteristics of the subjects in eligible studies

\begin{tabular}{|c|c|c|c|c|c|c|c|c|c|c|c|c|c|c|c|c|c|}
\hline \multirow[t]{3}{*}{ Study } & \multirow[t]{3}{*}{ Number Arms } & \multicolumn{4}{|c|}{ Efficacy } & \multicolumn{2}{|l|}{ Age } & \multirow{2}{*}{\multicolumn{2}{|c|}{$\frac{\text { Sex }}{\text { Male }}$}} & \multicolumn{4}{|c|}{ Disease status } & \multicolumn{4}{|c|}{ ECOG } \\
\hline & & $\overline{\mathrm{OS}}$ & PFS & TTP & ORR & Median & Range & & & \multicolumn{2}{|l|}{$\overline{L A}$} & \multicolumn{2}{|l|}{ ME } & \multicolumn{2}{|l|}{$0-1$} & \multicolumn{2}{|l|}{$\geq 2$} \\
\hline & & \multicolumn{6}{|c|}{ Median months } & $\mathrm{N}$ & $\%$ & $\mathrm{~N}$ & $\%$ & $\mathrm{~N}$ & $\%$ & $\mathrm{~N}$ & $\%$ & $\mathrm{~N}$ & 9 \\
\hline \multicolumn{18}{|c|}{ Fluoropyrimidine-based } \\
\hline \multirow[t]{2}{*}{ Ajani 2005} & 79 DTX+Cis+5-FU & 9.6 & 5.9 & NA & 43 & 57 & $21-83$ & 53 & 70 & 4 & 6 & 72 & 95 & 79 & 100 & 0 & 1 \\
\hline & $76 \mathrm{DTX}+\mathrm{Cis}$ & 10.5 & 5 & NA & 26 & 57 & $30-76$ & 61 & 77 & 1 & 1 & 75 & 95 & 75 & 99 & 0 & 1 \\
\hline \multirow[t]{2}{*}{ Douglass 1984} & 46 5-FU+Doxo+MMC & 29.5 & NA & NA & 39 & 61.0 & $32-81$ & 35 & 76 & 0 & 0 & 46 & 100 & 30 & 65 & 16 & 3 \\
\hline & 46 Doxo+MMC & 19 & NA & NA & 29 & 58 & $33-78$ & 37 & 80 & 0 & 0 & 46 & 100 & 28 & 61 & 18 & 3 \\
\hline \multirow[t]{2}{*}{ Roth 2007} & $41 \mathrm{DTX}+\mathrm{Cis}+5-\mathrm{FU}$ & 10.4 & NA & 4.6 & 36.6 & 61 & $35-78$ & 30 & 73 & 2 & 5 & 39 & 95 & 41 & 100 & 0 & 0 \\
\hline & $38 \mathrm{DTX}+\mathrm{Cis}$ & 11.0 & NA & 3.6 & 18.4 & 58 & $40-70$ & 29 & 76 & 7 & 18 & 31 & 82 & 38 & 100 & 0 & 0 \\
\hline \multirow[t]{2}{*}{ Van Cutsem 2015} & $89 \mathrm{DTX}+\mathrm{Ox}+5-\mathrm{FU}$ & 14.6 & 7.6 & NA & 46.6 & 58 & NA & 61 & 69 & 0 & 0 & 89 & 100 & 87 & 98 & 2 & 2 \\
\hline & 79 DTX+Ox & 8.93 & 4.5 & NA & 23.1 & 59 & NA & 51 & 65 & 0 & 0 & 79 & 100 & 77 & 99 & 1 & 1 \\
\hline \multicolumn{18}{|l|}{ Platinum-based } \\
\hline Kikuchi K 1990 & $32 \mathrm{ADM}+5-\mathrm{FU}+\mathrm{Cis}$ & NA & NA & NA & 6 & NA & NA & NA & NA & NA & NA & NA & NA & NA & NA & NA & \\
\hline & $33 \mathrm{ADM}+5-\mathrm{FU}$ & NA & NA & NA & 0 & NA & NA & NA & NA & NA & NA & NA & NA & NA & NA & NA & $N$ \\
\hline Park 2008 & $45 \mathrm{Cis}+\mid \mathrm{ri}+5-\mathrm{FU}$ & 10.5 & 6.2 & NA & 42 & 52 & $29-70$ & 30 & 76 & 0 & 0 & 45 & 100 & 38 & 84 & 7 & 1 \\
\hline & 46 Iri+5-FU & 10.7 & 4.8 & NA & 42 & 55 & $26-73$ & 30 & 67 & 0 & 0 & 45 & 100 & 35 & 78 & 11 & 2 \\
\hline Roth 1999 & $61 \mathrm{Epi}+\mathrm{Cis}+5-\mathrm{FU}$ & 9.6 & NA & NA & 42.6 & 54 & NA & 37 & 61 & 12 & 22 & 42 & 78 & 24 & 39 & 30 & 6 \\
\hline & $61 \mathrm{Epi+5-FU}$ & 7.1 & NA & NA & 28.6 & 56 & NA & 42 & 69 & 16 & 30 & 40 & 84 & 27 & 44 & 29 & 5 \\
\hline Anthracyclin-based & & & & & & & & & & & & & & & & & \\
\hline Douglass 1984 & 39 5-FU+Doxo+Me & 5.5 & NA & NA & 29 & 59.5 & $43-76$ & 28 & 71 & 0 & 0 & 39 & 100 & 30 & 77 & 9 & 2 \\
\hline & 48 5-FU+Me & 3.3 & NA & NA & 14 & 62.0 & $24-79$ & 38 & 80 & 0 & 0 & 48 & 100 & 35 & 72 & 13 & 2 \\
\hline Kim 2001 & $48 \mathrm{Epi}+\mathrm{Cis}+5-\mathrm{FU}$ & 8.5 & NA & 4.4 & 41.5 & 55 & NA & 45 & 75 & 3 & 5 & 57 & 95 & 54 & 90 & 6 & 1 \\
\hline & $48 \mathrm{Cis}+5-\mathrm{FU}$ & 7.3 & NA & 3.9 & 37.7 & 56 & NA & 42 & 70 & 3 & 5 & 57 & 95 & 53 & 88 & 7 & 1 \\
\hline KRGCGC 1992 & $25 \mathrm{Epi}+\mathrm{Cis}+5-\mathrm{FU}$ & 6.9 & NA & NA & 27 & 55 & NA & 45 & 75 & 3 & 5 & 57 & 97 & 54 & 90 & 6 & \\
\hline & $22 \mathrm{Cis}+5-\mathrm{FU}$ & 4 & NA & NA & 24 & 55 & NA & 45 & 75 & 3 & 5 & 57 & 95 & 54 & 90 & 6 & 1 \\
\hline Yun 2010 & 44 Epi+Cis+Cap & NA & 6.5 & NA & 37 & 55 & $37-51$ & 28 & 64 & NA & NA & NA & NA & 40 & 91 & 1 & 9 \\
\hline & 47 Cis+Cap & NA & 6.4 & NA & 38 & 58 & $33-75$ & 34 & 72 & NA & NA & NA & NA & 41 & 87 & 4 & 1 \\
\hline MC-based & & & & & & & & & & & & & & & & & \\
\hline Cullinan 1985 & 51 5-FU+Doxo+MMC & NA & NA & NA & 38.5 & 60 & NA & 39 & 76 & 20 & 39 & 31 & 61 & 32 & 63 & 19 & 3 \\
\hline & 49 Doxo+5-FU & NA & NA & NA & 27.7 & 63 & NA & 37 & 76 & 18 & 37 & 31 & 63 & 33 & 67 & 16 & 3 \\
\hline Koizumi 2004 & 33 5-DFUR+Cis+MMC & 8.03 & NA & NA & 25 & 58 & $36-79$ & 19 & 58 & NA & NA & NA & NA & 16 & 48 & 13 & 3 \\
\hline & 29 5-DFUR+Cis & 5.97 & NA & NA & 17.2 & 58 & $37-79$ & 17 & 59 & NA & NA & NA & NA & 25 & 86 & 6 & \\
\hline xane & & & & & & & & & & & & & & & & & \\
\hline Al-Batran 2013 & $79 \mathrm{DTX}+\mathrm{Ox}+5-\mathrm{FU}$ & 17.3 & 9.1 & NA & 48.6 & 69 & $65-81$ & 51 & 71 & 22 & 31 & 50 & 69 & 67 & 93 & 5 & 7 \\
\hline & $76 \mathrm{Ox}+5-\mathrm{FU}$ & 14.5 & 7.1 & NA & 28.17 & 70 & $65-82$ & 45 & 63 & 22 & 32 & 49 & 68 & 65 & 92 & 6 & $y$ \\
\hline Van Cutsem 2006 & $227 \mathrm{DTX}+\mathrm{Cis}+5-\mathrm{FU}$ & 9.2 & NA & 5.6 & 37 & 55 & $26-79$ & 159 & 72 & 6 & 3 & 213 & 96 & 218 & 99 & 3 & 1 \\
\hline & $230 \mathrm{Cis}+5-\mathrm{FU}$ & 8.6 & NA & 3.7 & 25 & 55 & $25-76$ & 158 & 71 & 6 & 3 & 217 & 97 & 211 & 99 & 3 & 1 \\
\hline Wang 2015 & $119 \mathrm{DTX}+\mathrm{Cis}+5-\mathrm{FU}$ & 10.2 & 7.2 & NA & 48.7 & 56.6 & $19-80$ & 81 & 68.1 & 30 & 25.2 & 89 & 77.4 & 115 & 96.6 & 4 & \\
\hline & $115 \mathrm{Cis}+5-\mathrm{FU}$ & 8.5 & 4.9 & NA & 33.9 & 55.5 & $33-74$ & 88 & 76.5 & 26 & 22.6 & 89 & 74.8 & 108 & 93.9 & 7 & \\
\hline Yamada 2018 & $370 \mathrm{~S}-1+\mathrm{Cis}$ & 15.3 & 6.5 & NA & 56 & NA & NA & NA & NA & NA & NA & NA & NA & NA & NA & NA & \\
\hline & 371 S-1+Cis+DOC & 14.2 & 7.4 & NA & 59.3 & NA & NA & NA & NA & NA & NA & NA & NA & NA & NA & NA & \\
\hline hotecan-based & & & & & & & & & & & & & & & & & \\
\hline Guimbaud 2014 & 209 Epi+Cis+Cape & 9.5 & 5.3 & NA & 39.2 & 61.4 & $28-84$ & 154 & 74 & 36 & 17 & 173 & 83 & 169 & 81 & 36 & \\
\hline & 207 5-FU+Iri & 9.7 & 5.8 & NA & 37.8 & 61.4 & $29-80$ & 155 & 75 & 31 & 15 & 176 & 85 & 173 & 84 & 27 & \\
\hline
\end{tabular}


Table 1 Characteristics of the subjects in eligible studies (Continued)

\begin{tabular}{|c|c|c|c|c|c|c|c|c|c|c|c|c|c|c|c|c|c|}
\hline \multirow[t]{3}{*}{ Study } & \multirow[t]{3}{*}{ Number Arms } & \multicolumn{4}{|c|}{ Efficacy } & \multicolumn{2}{|l|}{ Age } & \multirow{2}{*}{\multicolumn{2}{|c|}{$\frac{\text { Sex }}{\text { Male }}$}} & \multicolumn{4}{|c|}{ Disease status } & \multicolumn{4}{|c|}{ ECOG } \\
\hline & & OS & PFS & TTP & ORR & \multirow[t]{2}{*}{ Median } & \multirow[t]{2}{*}{ Range } & & & \multicolumn{2}{|l|}{ LA } & \multicolumn{2}{|l|}{ ME } & \multicolumn{2}{|l|}{$0-1$} & \multicolumn{2}{|l|}{$\geq 2$} \\
\hline & & \multicolumn{4}{|c|}{ Median months } & & & $\mathrm{N}$ & $\%$ & $\mathrm{~N}$ & $\%$ & $\mathrm{~N}$ & $\%$ & $\mathrm{~N}$ & $\%$ & $\mathrm{~N}$ & $\%$ \\
\hline \multirow[t]{2}{*}{ Lin 2009} & 13 5-FU+Ox+PTX & NA & NA & NA & 62.5 & 55 & $36-67$ & 18 & 72 & NA & NA & NA & NA & NA & NA & NA & NA \\
\hline & 12 5-FU+Iri & NA & NA & NA & 33.3 & 55 & $36-67$ & 18 & 72 & NA & NA & NA & NA & NA & NA & NA & \\
\hline \multicolumn{18}{|l|}{ Other } \\
\hline \multirow[t]{2}{*}{ Kim 1993} & 110 5-FU+Doxo+MMC & 6.84 & 3 & NA & 25 & 54 & $19-77$ & 68 & 62 & NA & NA & NA & NA & 75 & 68 & 23 & 21 \\
\hline & 112 Cis+5-FU & 8.61 & 5.5 & NA & 51 & 51 & $20-68$ & 71 & 63 & NA & NA & NA & NA & 83 & 74 & 20 & 18 \\
\hline \multirow[t]{2}{*}{ Li 2011} & $50 \mathrm{PTX}+\mathrm{Cis}+5-\mathrm{FU}$ & 10.8 & NA & NA & 48 & 59 & $20-74$ & 32 & 68 & 22 & 46 & 28 & 56 & NA & NA & NA & N \\
\hline & $44 \mathrm{Ox}+5-\mathrm{FU}$ & 9.9 & NA & NA & 45.5 & 58 & $20-75$ & 31 & 70 & 17 & 41 & 27 & 61 & NA & NA & NA & \\
\hline \multirow[t]{2}{*}{ Maiello 2011} & 36 Epi+Cis+Cap & NA & NA & NA & 54.3 & 58 & $39-74$ & 22 & 60 & NA & NA & NA & NA & NA & NA & NA & \\
\hline & $31 \mathrm{DTX}+5-\mathrm{FU}$ & NA & NA & NA & 22.6 & 61 & $44-75$ & 23 & 74 & NA & NA & NA & NA & NA & NA & NA & \\
\hline \multirow[t]{2}{*}{ Roth 2007} & $40 \mathrm{Epi}+\mathrm{Cis}+5-\mathrm{FU}$ & 8.3 & NA & 4.9 & 25 & 59 & $32-71$ & 30 & 75 & 7 & 17 & 33 & 83 & 40 & 100 & 0 & 0 \\
\hline & $41 \mathrm{DTX}+\mathrm{Cis}+5-\mathrm{FU}$ & 10.4 & NA & 4.9 & 36.6 & 61 & $35-78$ & 30 & 73 & 2 & 5 & 39 & 95 & 41 & 10 & 0 & 0 \\
\hline \multirow[t]{2}{*}{ Thuss-Patience 2005} & $45 \mathrm{Epi}+\mathrm{Cis}+5-\mathrm{FU}$ & 9.7 & NA & 5.5 & 35.6 & 63 & $33-75$ & 36 & 80 & 1 & 2 & 44 & 98 & 44 & 98 & 1 & 2 \\
\hline & $45 \mathrm{DTX}+5-\mathrm{FU}$ & 9.5 & NA & 5.3 & 37.8 & 62 & $34-75$ & 29 & 64 & 1 & 2 & 44 & 98 & 42 & 95 & 2 & 4 \\
\hline \multirow[t]{3}{*}{ Van Hoefer 2000} & 133 5-FU+Doxo+MTX & 6.7 & 3.3 & NA & 12 & 58 & $30-74$ & 96 & 72 & 22 & 17 & 111 & 83 & 117 & 88 & 16 & 12 \\
\hline & 134 Cis+5-FU & 7.2 & 4.1 & NA & 20 & 57 & $24-74$ & 91 & 68 & 21 & 16 & 113 & 84 & 114 & 85 & 20 & 15 \\
\hline & 132 Eto+5-FU+LV & 7.2 & 3.3 & NA & 9 & 59 & $25-74$ & 90 & 68 & 22 & 17 & 110 & 83 & 120 & 92 & 12 & 9 \\
\hline
\end{tabular}

OS Overall survival, PFS Progression-free survival, TTP Time to progression, ORR Objective response rate, LA Locally advanced, ME Metastatic disease, ECOGE Eastern Cooperative Oncology Group performance status, NA Not applicable, DTX Docetaxel, DOC Docetaxel, PTX Palictaxel,Ciscisplatin, 5- FU Fluorouracil, Cape Capcapecitabine, Cap Capcapecitabine, 5-DFUR Doxifluridine, Ox Oxaliplatin, Doxo Doxorubicin, Epi Epirubicin, Iri Irinotecan, MMC Mitomycin C, Eto Etoposide, Cis Cisplatin, ADM Adriamycin, Me Methyl-CCNU, S-1 Tegafur

\section{Anthracycline-based triplet versus non-anthracycline- based doublet chemotherapy}

For anthracycline-based regimens, four trials reported the comparison between anthracycline-based triplet chemotherapy and non-anthracycline-based doublet chemotherapy [29, 30, 33, 39]. The results of the subgroup analysis revealed that the addition of an anthracycline in triplet chemotherapy was not associated with a better OS than the doublet chemotherapy $(\mathrm{HR}=0.70 ; 95 \% \mathrm{CI}, 0.42-1.15 ; P=$ $0.16 ; \mathrm{I}^{2}=0 \%$, Additional file 1: Figure $\mathrm{S} 1$ ). Anthracyclinebased triplet chemotherapy was also not related to better ORR compared with doublet chemotherapy $\left(\mathrm{OR}=1.18 ; 95 \% \mathrm{CI}, 0.86-1.62 ; P=0.30 ; \mathrm{I}^{2}=0 \%\right.$, Additional file 3: Figure S3).

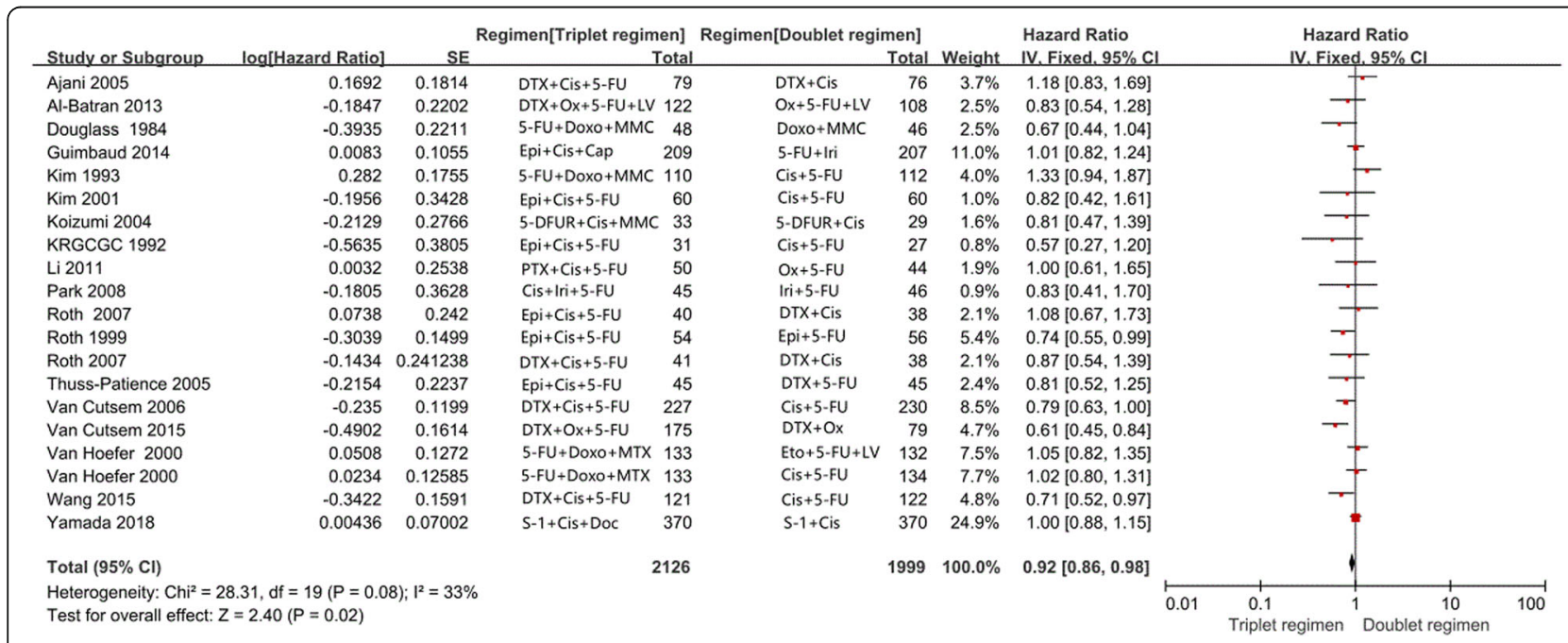

Fig. 2 Effects of triplet chemotherapy versus doublet chemotherapy on overall survival 


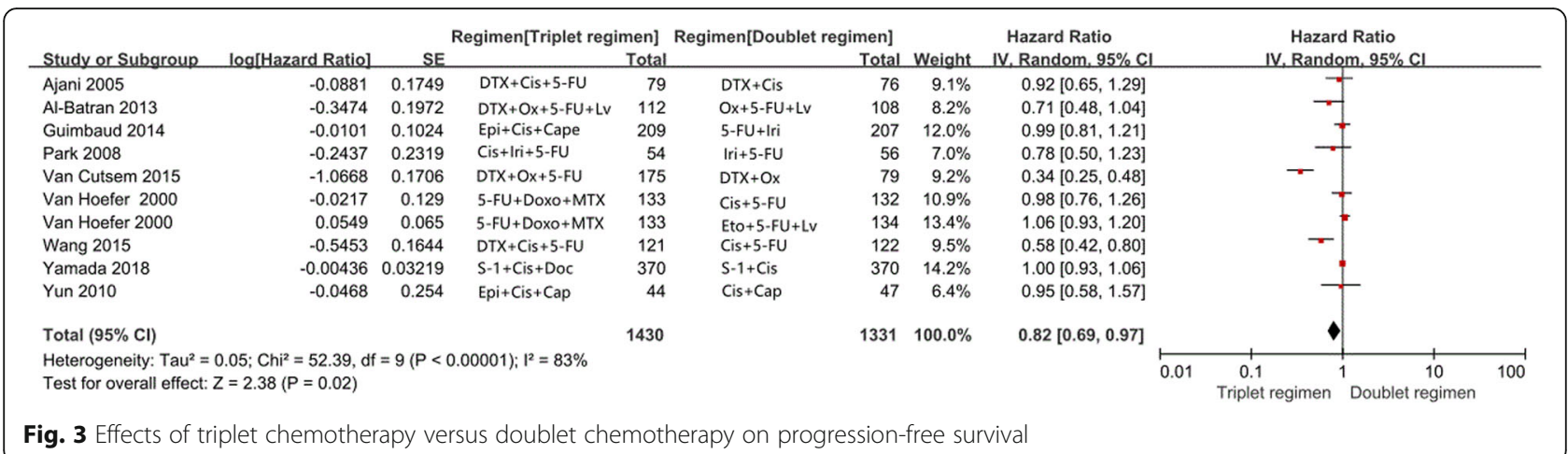

\section{Mitomycin-based triplet versus non-mitomycin-based doublet chemotherapy}

Two trials investigated the treatment difference between MMC-based triplet chemotherapy with nonMMC-based doublet chemotherapy [28, 32]. The results of the subgroup analysis revealed that MMCbased triplet chemotherapy had not an improvement on ORR compared with doublet chemotherapy (OR = 1.43; 95\% CI, 0.67-3.08; $P=0.36 ; \mathrm{I}^{2}=0 \%$, Additional file 3: Figure S3).

\section{Taxane-based triplet versus non-taxane-based doublet chemotherapy}

Four trials reported four taxane-based triplet chemotherapy compared with doublet chemotherapy [12, 14, 21, 26, 32]. The results of the subgroup analysis revealed that compared with taxane-based doublet chemotherapy, taxanebased triplet chemotherapy improved neither OS nor PFS $\left(\mathrm{HR}=0.91 ; 95 \% \mathrm{CI}, 0.81-1.01 ; P=0.07 ; \mathrm{I}^{2}=50 \%\right.$ vs. HR $=$ 0.76; 95\% CI, 0.52-1.11; $P=0.16 ; \mathrm{I}^{2}=85 \%$, respectively, Additional file 1: Figure S1, Additional file 2: Figure S2). However, taxane-based triplet chemotherapy improved significantly the ORR $(\mathrm{OR}=1.22 ; 95 \% \mathrm{CI}, 1.10-1.36 ; P=$ $0.0002 ; \mathrm{I}^{2}=75 \%$, Additional file 3: Figure S3).

\section{Irinotecan-based triplet versus non-irinotecan-based doublet chemotherapy}

Considering there was no study comparing irinotecanbased triplet regimens with non-irinotecan-based doublet regimen, there were actually two trials that compared irinotecan-based doublet chemotherapy with irinotecan- based triplet chemotherapy regimens [22, 35], and the subgroup analysis also estimated the different treatment outcomes between the two groups, although the chemotherapeutic drugs in doublet regimens are not identical to triplet regimens. The results of the subgroup analysis revealed that triplet chemotherapy regimens did not improve the ORR $(\mathrm{OR}=1.08 ; 95 \% \mathrm{CI}, 0.85-1.37 ; P=0.55$; $\mathrm{I}^{2}=31 \%$, Additional file 3: Figure S3).

\section{Other triplet versus non-doublet chemotherapies}

Eight trials compared other triplet chemotherapies with doublet chemotherapies. Subgroup analysis indicated that triplet chemotherapy did not improve both OS and PFS compared with doublet chemotherapy $(\mathrm{HR}=1.05$; 95\% CI, $0.92-1.21 ; P=0.46 ; \mathrm{I}^{2}=0 \%$ vs. $\mathrm{HR}=1.04 ; 95 \%$ CI, $0.93-1.17 ; P=0.50 ; \mathrm{I}^{2}=0 \%$, respectively, Additional file 1: Figure S1, Additional file 2: Figure S2). Moreover, triplet chemotherapy had lower ORR than doublet chemotherapy $(\mathrm{HR}=0.95 ; 95 \% \mathrm{CI}, 0.76-1.19 ; P=0.66$; $\mathrm{I}^{2}=63 \%$; Additional file 3: Figure S3).

\section{Asian and Western patients}

A total of 11 and 10 trials were conducted in Asian and Western patients, respectively. Two other trials were analyzed individually as the included patients were both from Asia and the Western, but detailed geographic data of these patients were not taken. Subgroup metaanalyses based on different patients including Asians and Westerners were further performed (Fig. 6). The results revealed that triplet chemotherapy did not improve OS compared with the doublet chemotherapy $(\mathrm{HR}=$

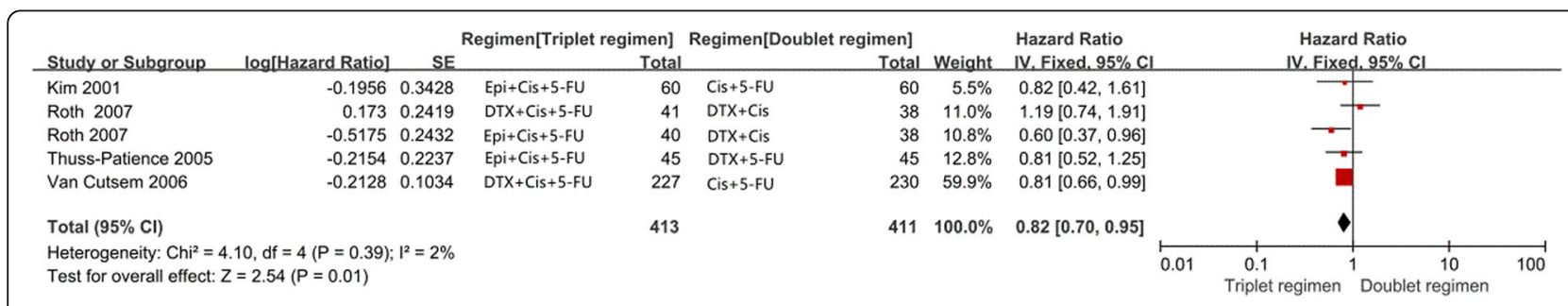

Fig. 4 Effects of triplet chemotherapy versus doublet chemotherapy on time to progress 


\begin{tabular}{|c|c|c|c|c|c|c|c|c|c|c|c|}
\hline \multicolumn{4}{|c|}{ Regimen[Triplet regimen] } & \multicolumn{2}{|c|}{ Regimen[Doublet regimen] } & & \multirow{2}{*}{$\begin{array}{c}\text { Risk Ratio } \\
\text { M-H. Fixed, } 95 \% \mathrm{Cl}\end{array}$} & \multirow{2}{*}{\multicolumn{4}{|c|}{$\begin{array}{c}\text { Risk Ratio } \\
\text { M-H. Fixed. } 95 \% \mathrm{Cl} \\
\end{array}$}} \\
\hline Study or Subgroup & \multicolumn{2}{|c|}{ Events } & \multirow{2}{*}{$\begin{array}{r}\text { Total } \\
79\end{array}$} & Events & Total & Total Weight & & & & & \\
\hline Ajani 2005 & $\mathrm{DTX}+\mathrm{Cis}+5-\mathrm{Fu}$ & 34 & & $D T X+C$ is & 76 & $3.1 \%$ & $1.64[1.04,2.57]$ & & & 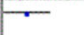 & \\
\hline Al-Batran 2013 & $D T X+O x+5-F U+L V$ & 35 & 62 & $O x+5-F U+L V 2$ & 68 & $2.9 \%$ & $1.92[1.25,2.95]$ & & & - & \\
\hline Cullinan 1985 & 5-FU+Doxo+MMC & 5 & 13 & Doxo+5.Fu & 11 & $0.5 \%$ & $1.41[0.43,4.61]$ & & & & \\
\hline Douglass 1984 & $5-\mathrm{FU}+\mathrm{Doxo}+\mathrm{Me}$ & 11 & 39 & $5-F U+M e$ & 44 & $0.8 \%$ & $2.07[0.84,5.07]$ & & & & \\
\hline Douglass 1984 & $5-F U+D O X 0+M M C$ & $c 18$ & 46 & Doxo+MMC 1 & 46 & $2.0 \%$ & $1.38[0.77,2.48]$ & & & . & \\
\hline Guimbaud 2014 & Epi+Cis + Cap & 74 & 189 & 5-FU+ lri 7 & 198 & $11.0 \%$ & $1.03[0.80,1.33]$ & & & 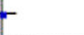 & \\
\hline Kikuchi K 1990 & $A D M+5-F U+C$ is & 6 & 32 & $A D M+5-F U$ & 33 & $0.1 \%$ & $13.39[0.79,228.40]$ & & & & \\
\hline Kim 1993 & $\mathrm{FU}+\mathrm{DoxO}+\mathrm{MMC}$ & 14 & 57 & $\mathrm{Cis}+5-\mathrm{Fu} \quad 2$ & 55 & $4.3 \%$ & $0.48[0.29,0.81]$ & & & & \\
\hline Kim 2001 & Epi $+\mathrm{Cis}+5$-FU & 22 & 53 & Cist5-Fu 2 & 53 & $3.0 \%$ & $1.10[0.69,1.76]$ & & & $\leftarrow$ & \\
\hline Koizumi 2004 & 5- DFUR+Cis+MMC & c 8 & 32 & 5-DFUR + Cis & 29 & $0.8 \%$ & $1.45[0.53,3.93]$ & & & & \\
\hline KRGCGC 1992 & Epi+Cis+5-FU & 7 & 25 & $\mathrm{Cis}+5-\mathrm{FU}$ & 22 & $0.8 \%$ & $1.23[0.46,3.33]$ & & & . & \\
\hline Li 2011 & $\mathrm{PTX}+\mathrm{Cis}+5-\mathrm{Fu}$ & 24 & 50 & $O x+5-F u$ & 44 & $3.2 \%$ & $1.06[0.68,1.63]$ & & & $\leftarrow$ & \\
\hline Lin 2009 & 5-FU+OX+PTX & 8 & 13 & S-Fu+lri & 12 & $0.6 \%$ & $1.85[0.74,4.58]$ & & & & \\
\hline Maiello 2011 & Epi+Cis+Cap & 20 & 36 & $D T X+5-F U$ & 31 & $1.1 \%$ & $2.46[1.20,5.03]$ & & & & \\
\hline Park 2008 & Cistlri+5-fu & 19 & 45 & $\mid r i+5-F u$ & 45 & $2.9 \%$ & $1.00[0.62,1.62]$ & & & F & \\
\hline Roth 2007 & Epi+Cis $+5-F U$ & 10 & 40 & $D T X+C$ is & 38 & $1.1 \%$ & $1.36[0.58,3.20]$ & & & & \\
\hline Roth 1999 & Epi + Cis +5 -Fu & 23 & 54 & Epi+5-FU 1 & 56 & $2.4 \%$ & $1.49[0.89,2.50]$ & & & - & \\
\hline Roth 2007 & $\mathrm{DTX}+\mathrm{Cis}+5-\mathrm{FU}$ & 15 & 41 & $\mathrm{DTX}+\mathrm{C}$ is & 38 & $1.1 \%$ & $1.99[0.91,4.34]$ & & & & \\
\hline Thuss-Patience 200 & 05 Epi+Cist+5-Fu & 16 & 43 & DTX+5-FU $\quad 1$ & 43 & $2.6 \%$ & $0.94[0.55,1.61]$ & & & - & \\
\hline Van Cutsem 2006 & $\mathrm{DTX}+\mathrm{Cis}+5-\mathrm{FU}$ & 81 & 221 & Cis +5 -Fu 5 & 224 & $8.5 \%$ & $1.44[1.08,1.91]$ & & & - & \\
\hline Van Cutsem 2015 & $\mathrm{DTX}+\mathrm{Ox}+5=\mathrm{FU}$ & 62 & 170 & $D T X+O X$ & 78 & $3.7 \%$ & $1.58[1.01,2.48]$ & & & & \\
\hline Van Hoefer 2000 & 5-FU+Doxo+MTX & 10 & 85 & Cist +5 -Fu 1 & 81 & $2.5 \%$ & $0.60[0.29,1.23]$ & & & - & \\
\hline Van Hoefer 2000 & $5-F U+D o x o+M T X$ & 10 & 85 & Eto $+5-F U+L V$ & 79 & $1.1 \%$ & $1.33[0.53,3.32]$ & & & & \\
\hline Wang 2015 & $\mathrm{DTX}+\mathrm{Cis}+5-\mathrm{Fu}$ & 60 & 119 & Cis +5 - Fu 4 & 115 & $6.1 \%$ & $1.45[1.07,1.97]$ & & & - & \\
\hline Yamada 2018 & $s-1+C i s+D o c \quad 2$ & 219 & 370 & $\mathrm{~S}-1+\mathrm{C}$ is $\quad 20$ & 371 & $31.3 \%$ & $1.06[0.93,1.19]$ & & & & \\
\hline Yun 2010 & $\mathrm{Epi}+\mathrm{Cis}+\mathrm{Cap}$ & 16 & 44 & $\mathrm{Cis}+\mathrm{Cap}$ & 45 & $2.5 \%$ & $0.96[0.56,1.65]$ & & & & \\
\hline Total $(95 \% \mathrm{Cl})$ & & & 2043 & & 1935 & $100.0 \%$ & $1.21[1.12,1.31]$ & & & 1 & \\
\hline Total events & & 827 & & & 355 & & & & & & \\
\hline $\begin{array}{l}\text { Heterogeneity: } \mathrm{Ch}^{2} \\
\text { Test for overall effec }\end{array}$ & $\begin{array}{l}2=46.00, d f=25 \\
\text { t: } Z=4.68(P<\end{array}$ & $\begin{array}{l}5(P= \\
<0.00\end{array}$ & $I^{2}=46$ & & & & & & $\begin{array}{cc}0.1 & 1\end{array}$ & 1 & $\begin{array}{l}10 \\
\text { imen }\end{array}$ \\
\hline
\end{tabular}

Fig. 5 Effect of triplet chemotherapy versus doublet chemotherapy on objective response rate

\begin{tabular}{|c|c|c|c|c|c|c|c|c|c|c|c|}
\hline \multirow{2}{*}{$\begin{array}{l}\text { Study or Subgroup } \\
\text { 1.3.1 Asian }\end{array}$} & \multirow{2}{*}{ logiHazard Ratiol_ } & \multicolumn{3}{|c|}{ Regimen[Triplet regimen] } & \multicolumn{2}{|c|}{ Regimen[Doublet regimen] } & \multicolumn{2}{|c|}{$\begin{array}{l}\text { Hazard Ratio } \\
\text { Weight } \\
\text { IV Fixed, } 95 \% \mathrm{Cl}\end{array}$} & \multicolumn{3}{|c|}{$\begin{array}{l}\text { Hazard Ratio } \\
\text { IV Fixed.95\% CI }\end{array}$} \\
\hline & & & & & & & & & & & \\
\hline Kim 1993 & 0.282 & 0.1755 & 5. $F U+D o \times O+M M C$ & 110 & Cis+5-FU & 112 & $4.0 \%$ & $1.33[0.94,1.87]$ & & - & \\
\hline Kim 2001 & -0.1956 & 0.3428 & Epi+Cis+5-FU & 60 & $\mathrm{Cis}+5 \cdot \mathrm{Fu}$ & 60 & $1.0 \%$ & $0.82\{0.42,1.61\}$ & & & \\
\hline Kolzumi 2004 & -0.2129 & 0.2766 & 5-DFUR+Cis+MMC & 33 & 5-DFUR +Cis & 29 & $1.6 \%$ & $0.81[0.47,1.39]$ & & & \\
\hline KRGCGC 1992 & -0.5635 & 0.3805 & $\mathrm{Epi}+\mathrm{Cis}+5-\mathrm{FU}$ & 31 & Cis $+5-F U$ & 27 & $0.8 \%$ & $0.57[0.27,1.20]$ & & & \\
\hline Li 2011 & 0.0032 & 0.2538 & $\mathrm{PTX}+\mathrm{C}$ is $+5=\mathrm{FU}$ & 50 & $\mathrm{OX}+5-\mathrm{Fu}$ & 44 & $1.9 \%$ & $1.00[0.61,1.65]$ & & & \\
\hline Park 2008 & $\begin{array}{l}0.0002 \\
-0.1805\end{array}$ & 0.3628 & 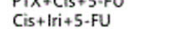 & 45 & $\begin{array}{l}\text { Irit+5-FU } \\
\text { InU }\end{array}$ & 46 & $0.9 \%$ & $0.83[0.41,1.70]$ & & & \\
\hline Wang 2015 & -0.3422 & 0.1591 & $\mathrm{DTX}+\mathrm{Cis}+5$-FU & 121 & Cis $+5-\mathrm{Fu}$ & 122 & $4.8 \%$ & $0.71[0.52,0.97]$ & & & \\
\hline Yamada 2018 & 0.00436 & 0.07002 & $\mathrm{~S}-1+\mathrm{Cis}_{\mathrm{is}}+\mathrm{Doc}$ & 370 & $\mathrm{~S}-1+\mathrm{C}$ is & 370 & $24.9 \%$ & $1.00[0.88,1.15]$ & 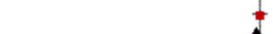 & 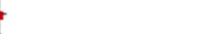 & \\
\hline Subtotal $(95 \% \mathrm{Cl})$ & & & & 820 & & 810 & $39.9 \%$ & $0.96[0.86,1.07]$ & 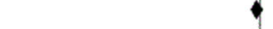 & & \\
\hline \multicolumn{12}{|c|}{$\begin{array}{l}\text { Heterogeneity: } \text { Chi }^{2}=10.04, \text { df }=7(P=0.19) ; p^{2}=30 \% \\
\text { Test for overall effect: } Z=0.73(P=0.47)\end{array}$} \\
\hline \multicolumn{12}{|l|}{ 1.3.2 Westem } \\
\hline Al-Batran 2013 & -0.1847 & 0.2202 & $D T X+O x+5-F U+L V$ & 122 & $\mathrm{Ox}+5-\mathrm{FU}+\mathrm{LV}$ & 108 & $2.5 \%$ & $0.83[0.54,1.28]$ & $\rightarrow$ & & \\
\hline Douglass 1984 & -0.3935 & 0.2211 & $5-F U+D o x o+M M C$ & 48 & Doxo+MMC & 46 & $2.5 \%$ & $0.67[0.44,1.04]$ & & & \\
\hline Guimbaud 2014 & 0.0083 & 0.1055 & Epi+Cis+Cap & 209 & 5-FU+liri & 207 & $11.0 \%$ & $1.01[0.82,1.24]$ & & & \\
\hline Roth 2007 & 0.0738 & 0.242 & $\mathrm{Epi}+\mathrm{Cis}+5$-Fu & 40 & $\mathrm{DTX}+\mathrm{Cis}$ & 38 & $2.1 \%$ & $1.08[0.67,1.73]$ & 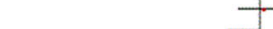 & & \\
\hline Roth 1999 & -0.3039 & 0.1499 & $\mathrm{Epi}+\mathrm{Cis}+5-\mathrm{FU}$ & 54 & Epi+5-FU & 56 & $5.4 \%$ & $0.74[0.55,0.99]$ & $\rightarrow$ & & \\
\hline Roth 2007 & -0.1434 & 0.241238 & $\mathrm{Epi}+\mathrm{Cis}+5$-Fu & 41 & $\mathrm{DTX}+\mathrm{Cis}$ & 38 & $2.1 \%$ & $0.87[0.54,1.39]$ & & & \\
\hline Thuss-Patience 2005 & .0 .2154 & 0.2237 & Epi+Cis +5-Fu & 45 & DTX $+5-F U$ & 45 & $2.4 \%$ & $0.81[0.52,1.25]$ & $\longrightarrow$ & & \\
\hline Van Cutsem 2015 & -0.4902 & 0.1614 & $D T X+O x+5-F U$ & 175 & $\mathrm{DTX}+\mathrm{Ox}+5-\mathrm{FU}$ & 79 & $4.7 \%$ & $0.61[0.45,0.84]$ & - & & \\
\hline Van Hoefer 2000 & 0.0508 & 0.1272 & 5-FU+Doxo+MTX & 133 & Eto $+5-F U+L V$ & 132 & $7.5 \%$ & $1.05[0.82,1.35]$ & & & \\
\hline Van Hoefer 2000 & 0.0234 & 0.12585 & 5-FU+Doxa+MTX & 133 & Cist5-Fu & 134 & $7.7 \%$ & $1.02[0.80,1.31]$ & & & \\
\hline Subtotal $(95 \% \mathrm{Cl}$ ) & & & & 1000 & & 883 & $47.9 \%$ & $0.89[0.81,0.99]$ & 4 & & \\
\hline \multicolumn{12}{|c|}{$\begin{array}{l}\text { Heterogeneity: } C \mathrm{Ch}^{P}=13.78, \text { df }=9(P=0.13) ; P=35 \% \\
\text { Test for overall effect: } Z=2.24(P=0.03)\end{array}$} \\
\hline \multicolumn{12}{|l|}{ 1.3.3 Western+Taiwan } \\
\hline Ajani 2005 & 0.1692 & 0.1814 & $\mathrm{DTX}+\mathrm{Cis}+5-\mathrm{Fu}$ & 79 & $\mathrm{DTX}+\mathrm{C}$ is & 76 & $3.7 \%$ & 1. $18[0.83,1.69]$ & & - & \\
\hline Van Cutsem 2006 & -0.235 & 0.1199 & $\mathrm{OTX}+\mathrm{Cis}+5-\mathrm{FU}$ & 227 & $\mathrm{Cis}+5-\mathrm{FU}$ & 230 & $8.5 \%$ & $0.79[0.63,1.00]$ & & & \\
\hline Subtotal (95\% Cl) & & & & 306 & & 306 & $12.2 \%$ & $0.89[0.73,1.09]$ & 4 & & \\
\hline \multicolumn{12}{|c|}{$\begin{array}{l}\text { Heterogeneity: } \mathrm{Ch}^{2} \mathrm{P}=3.46, d f=1 \quad(P=0.06): 1^{2}=71 \% \\
\text { Test for overall etfect: } Z=1.12(P=0.26)\end{array}$} \\
\hline \multirow{2}{*}{\multicolumn{2}{|c|}{$\begin{array}{l}\text { Total }(95 \% \mathrm{Cl}) \\
\text { Heterogeneity: } \mathrm{Ch}=28.31, \mathrm{df}=19(\mathrm{P}=0.08) \\
\text { Test for overall effect: } Z=2.40(\mathrm{P}=0.02) \\
\text { Test for subaroud differences: } \mathrm{Ch} \mathrm{P}^{2}=1.04 \text {. di }=\end{array}$}} & & & 2126 & & 1999 & $100.0 \%$ & $0.92[0.86,0.98]$ & $t$ & & \\
\hline & & $\begin{array}{l}8)::^{2}=33 \% \\
=2(P=0.6\end{array}$ & $60 \%, p=0 \%$ & & & & & & $\begin{array}{ccc}0.01 & 0.1 \\
& \text { Favours [Triplet regimen] }\end{array}$ & $\frac{1}{10}$ & 100 \\
\hline
\end{tabular}


0.96; 95\% CI, 0.86-1.07; $P=0.47 ; \mathrm{I}^{2}=30 \%$ ) among Asian patients. However, triplet chemotherapy significantly improved OS compared with the doublet chemotherapy (HR $=0.89 ; 95 \%$ CI, $\left.0.81-0.99 ; P=0.03 ; \mathrm{I}^{2}=35 \%\right)$ among Western patients.

\section{Comparison of the same chemotherapy regimens}

This meta-analysis included a lot of primary studies compared different doublet and triplet chemotherapy. Considering that the inherent heterogeneity of different chemotherapeutic drugs may affect the results of this metaanalysis, we choose the same chemotherapy regimens between triplet and doublet chemotherapy to carry out subgroup meta-analysis, and studies that have only one type of triplet and doublet chemotherapy regimens were deleted. The results of the subgroup analysis revealed that triplet chemotherapy regimens improve the $\mathrm{OS}(\mathrm{OR}=0.88 ; 95 \%$ CI, $0.80-0.97 ; P=0.009 ; \mathrm{I} 2=48 \%$, Additional file 4: Figure S4) and $\operatorname{ORR}(\mathrm{OR}=1.26 ; 95 \% \mathrm{CI}, 1.15-1.39 ; \quad P<0.00001$; I $2=50 \%$, Additional file 6: Figure S6), and PFS has not been further improved $(\mathrm{OR}=0.67 ; 95 \% \mathrm{CI}, 0.45-1.00 ; P<0.00001$; I $2=92 \%$, Additional file 5: Figure S5).

\section{Publication bias}

The funnel plots did not show significant asymmetry for triplet versus doublet chemotherapy in terms of OS, PFS, TTP, and ORR (Fig. 7).

\section{Toxicities}

Main data were available for 5 hematological, 16 nonhematological, and 4 laboratory-assessed items among the 23 trials. We summarized grade $1-2$ and grade $3-4$ AEs, and the results are shown in Table 2. The most common grade 3-4 hematological toxicities were neutropenia and leucopenia, while the most common nonhematological toxicities were nausea, vomiting, diarrhea, stomatitis, anorexia, fatigue, alopecia, and lethargy. There were significantly more incidences of grade 3-4 neutropenia $(\mathrm{RR}=1.46 ; 95 \% \mathrm{CI}, 1.32-1.60 ; P<0.001)$, leucopenia $(\mathrm{RR}=1.51 ; \quad 95 \% \quad \mathrm{CI}, \quad 1.33-1.71 ; \quad P<0.001), \quad$ febrile

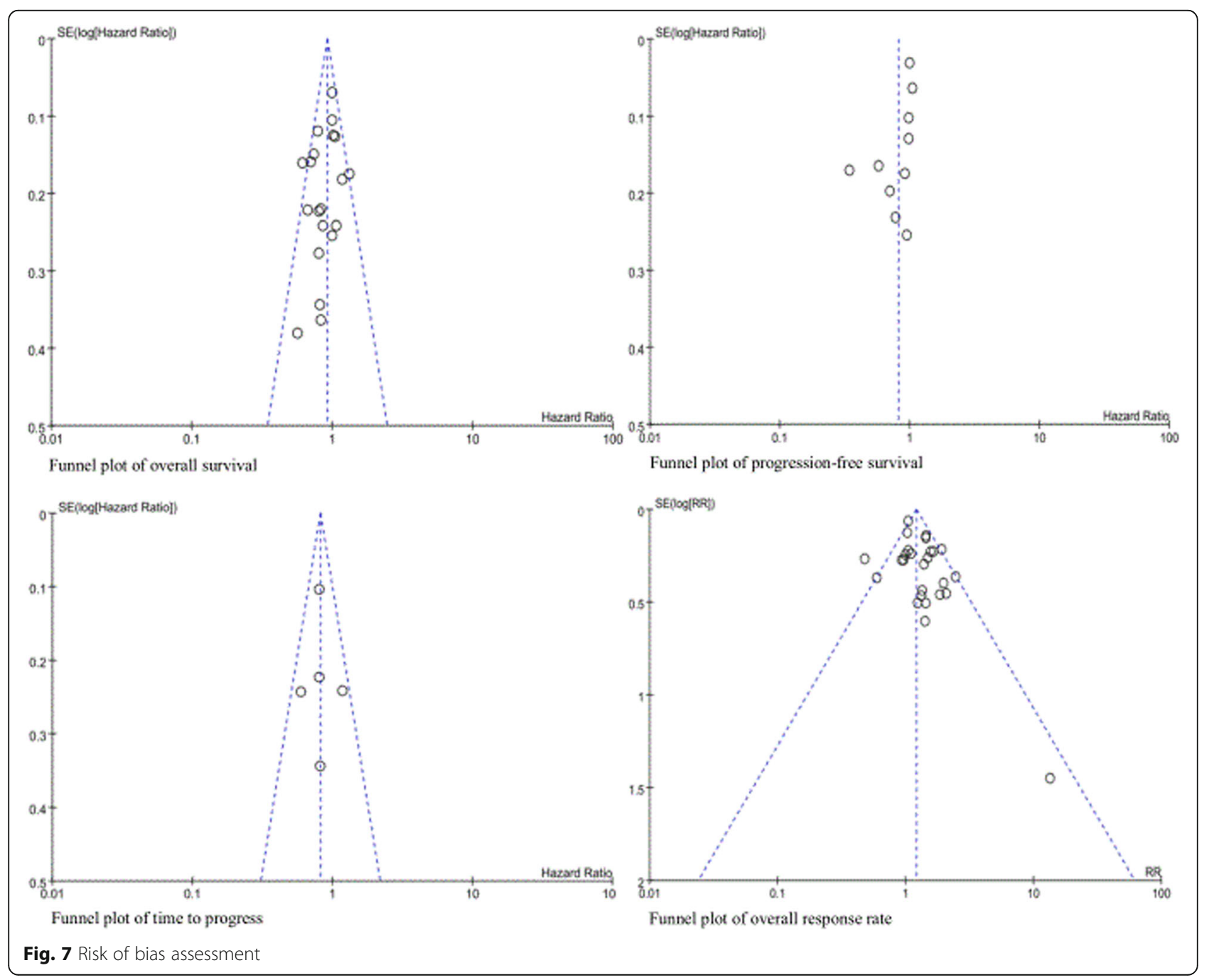


Table 2 Toxicity results of triplet chemotherapy compared with doublet chemotherapy

\begin{tabular}{|c|c|c|c|c|c|c|c|c|c|c|c|c|c|c|c|c|}
\hline \multirow[t]{3}{*}{ Toxicity Category } & \multicolumn{8}{|c|}{ Grade 1 or 2} & \multicolumn{8}{|c|}{ Grade 3 or 4} \\
\hline & \multicolumn{3}{|c|}{ Triplet } & \multicolumn{3}{|c|}{ Doublet } & \multirow[b]{2}{*}{ RR } & \multirow[b]{2}{*}{$95 \% \mathrm{Cl}$} & \multicolumn{3}{|c|}{ Triplet } & \multicolumn{3}{|c|}{ Doublet } & \multirow[b]{2}{*}{ RR } & \multirow[b]{2}{*}{$95 \% \mathrm{Cl}$} \\
\hline & $\mathrm{T}$ & Total & $\%$ & $\mathrm{~T}$ & Total & $\%$ & & & $\mathrm{~T}$ & Total & $\%$ & $\mathrm{~T}$ & Total & $\%$ & & \\
\hline \multicolumn{17}{|l|}{ Hematological } \\
\hline Neutropenia & 131 & 748 & 18 & 184 & 651 & 28 & 0.62 & $0.51-0.76$ & 682 & 1234 & 55 & 368 & 970 & 38 & 1.46 & $1.32-1.60$ \\
\hline Leucopenia & 291 & 680 & 42 & 277 & 684 & 40 & 1.06 & $0.93-1.20$ & 474 & 1102 & 43 & 252 & 885 & 28 & 1.51 & $1.33-1.71$ \\
\hline Anemia & 498 & 925 & 53 & 454 & 924 & 49 & 1.10 & $1.00-1.20$ & 111 & 784 & 14 & 123 & 682 & 18 & 0.79 & $0.62-0.99$ \\
\hline Thrombocytopenia & 151 & 846 & 18 & 162 & 848 & 19 & 0.93 & $0.76-1.14$ & 113 & 1181 & 9 & 119 & 934 & 12 & 0.75 & $0.59-0.96$ \\
\hline Febrile neutropenia & 79 & 254 & 31 & 46 & 214 & 21 & 1.45 & $1.06-1.98$ & 109 & 1026 & 10 & 44 & 774 & 6 & 1.87 & $1.33-2.62$ \\
\hline \multicolumn{17}{|l|}{ Non-hematological } \\
\hline Nausea & 358 & 584 & 61 & 353 & 491 & 71 & 0.85 & $0.78-0.93$ & 115 & 1130 & 10 & 118 & 900 & 13 & 0.78 & $0.61-0.99$ \\
\hline Vomiting & 295 & 716 & 41 & 275 & 622 & 44 & 0.93 & $0.82-1.06$ & 74 & 795 & 9 & 70 & 618 & 11 & 0.82 & $0.60-1.12$ \\
\hline Diarrhea & 343 & 748 & 46 & 201 & 651 & 31 & 1.49 & $1.29-1.71$ & 125 & 1590 & 8 & 62 & 1323 & 5 & 1.68 & $1.25-2.25$ \\
\hline Stomatitis & 254 & 716 & 35 & 165 & 622 & 27 & 1.34 & $1.14-1.58$ & 151 & 1594 & 9 & 96 & 923 & 10 & 0.91 & $0.71-1.16$ \\
\hline Anorexia & 150 & 467 & 32 & 138 & 376 & 36 & 0.88 & $0.73-1.06$ & 57 & 546 & 10 & 41 & 452 & 9 & 1.15 & $0.79-1.69$ \\
\hline Fatigue & 175 & 376 & 46 & 132 & 283 & 47 & 1.00 & $0.85-1.18$ & 52 & 376 & 14 & 35 & 283 & 12 & 1.12 & $0.75-1.67$ \\
\hline Hand foot yndrome & 45 & 259 & 17 & 32 & 168 & 19 & 0.91 & $0.61-1.37$ & 17 & 376 & 5 & 7 & 237 & 3 & 1.53 & $0.64-3.64$ \\
\hline Sensory europathy & 227 & 597 & 38 & 162 & 507 & 32 & 1.19 & $1.01-1.40$ & 75 & 1021 & 7 & 49 & 805 & 6 & 1.21 & $0.85-1.71$ \\
\hline Alopecia & 83 & 242 & 34 & 39 & 148 & 26 & 1.30 & $0.94-1.97$ & 87 & 538 & 16 & 45 & 274 & 16 & 0.98 & $0.71-1.37$ \\
\hline Pigmentation & 33 & 215 & 15 & 11 & 123 & 9 & 1.72 & $0.90-3.27$ & 8 & 296 & 3 & 7 & 161 & 4 & 0.62 & $0.23-1.68$ \\
\hline Lethargy & 83 & 221 & 37 & 74 & 224 & 33 & 1.14 & $0.88-1.46$ & 57 & 300 & 19 & 45 & 300 & 15 & 1.27 & $0.89-1.81$ \\
\hline Infected & 21 & 293 & 7 & 22 & 294 & 7 & 0.96 & $0.54-1.70$ & 41 & 291 & 14 & 23 & 294 & 78 & 1.80 & $1.11-2.92$ \\
\hline Constipation & 62 & 286 & 22 & 41 & 193 & 21 & 1.02 & $0.72-1.45$ & 0 & 286 & 0 & 5 & 193 & 0.06 & 0.06 & $0.00-1.10$ \\
\hline Fluid retention & 17 & 72 & 24 & 10 & 70 & 14 & 1.65 & $0.81-3.36$ & 1 & 72 & 1.3 & 3 & 70 & 4 & 0.32 & $0.03-3.04$ \\
\hline Allergy & 5 & 72 & 7 & 5 & 70 & 7 & 0.97 & $0.29-3.21$ & 1 & 72 & 1.3 & 1 & 70 & 1.3 & 0.97 & $0.06-15.2$ \\
\hline Abdominal pain & 33 & 170 & 19 & 21 & 78 & 27 & 0.72 & $0.45-1.16$ & 6 & 170 & 3.5 & 4 & 78 & 5.1 & 0.69 & $0.20-2.37$ \\
\hline \multicolumn{17}{|l|}{ Laboratory-assessed items } \\
\hline Increased ALT & 28 & 149 & 19 & 28 & 144 & 19 & 0.97 & $0.60-1.55$ & 0 & 149 & 0 & 1 & 144 & 0.6 & 0.32 & $0.01-7.85$ \\
\hline Increased AST & 35 & 104 & 34 & 21 & 99 & 21 & 1.59 & $1.00-2.53$ & 0 & 104 & 0 & 1 & 99 & 1 & 0.32 & $0.01-7.70$ \\
\hline Increased ALP & 24 & 72 & 33 & 27 & 70 & 38 & 0.86 & $0.56-1.34$ & 1 & 72 & 1.3 & 2 & 70 & 2.8 & 0.49 & $0.05-1.40$ \\
\hline Creatinine & 18 & 149 & 12 & 26 & 144 & 18 & 0.67 & $0.38-1.17$ & 1 & 149 & 0.6 & 0 & 144 & 0 & 2.90 & $0.12-7.61$ \\
\hline
\end{tabular}

ALT Alanine aminotransferase, AST Aspartate aminotransferase, ALP alkaline phosphatase

neutropenia $(\mathrm{RR}=1.87 ; 95 \% \mathrm{CI}, 1.33-2.62 ; P<0.001)$, diarrhea $(\mathrm{RR}=1.68 ; 95 \% \mathrm{CI}, 1.25-2.25 ; P<0.001)$, and infection $(\mathrm{RR}=1.80 ; 95 \% \mathrm{CI}, 1.11-2.92 ; P=0.02)$ in triplet chemotherapy group compared with combination chemotherapy group, while equivalent frequencies of grade 3-4 AEs were found between the two groups.

\section{Discussion}

The debate of triplet or doublet chemotherapy in treating patients with advanced gastric cancer has been existing for a long time, which started from the 1980s. Most of the earliest studies of triplet and doublet chemotherapy contained drugs, such as FU, Doxo, MMC, and Eto. With the development of the novel chemotherapeutic drugs, triplet and doublet chemotherapy regimens contained additional new drugs such as Epi, Iri, Taxa, Cap, Ox, and $\mathrm{T}$ in triplet or doublet chemotherapy in treating advanced gastric cancer.

Though nearly 30 RCTs were conducted, whether triplet or doublet chemotherapy improves the survival of patients with advanced gastric cancer remains unclear. The results were also identical among meta-analyses [8, 9, 41]. TTP in all patients with advanced gastric cancer. The result of OS and PFS was in line with the previous meta-analyses [9]. We enrolled all RCTs from the 1980s to October, 2018 and strictly and separately finished pooled analysis of PFS and TTP among 23 trials. A previous meta-analysis emulates PFS and TTP together [9]. Considering the difference of definition and clinical significance, pooled TTP analysis was individually made among included trials. Triplet 
regimens were in favor of longer TTP compared with doublet chemotherapy. Additionally, as expected, triplet regimens could result to a higher ORR than doublet regimens.

Fluorouracil-based, platinum-based, MMC-based, and anthracycline-based chemotherapies were the early regimens in treating patients with treatment-naive advanced gastric cancer in RCTs [28, 29]. The common doublet regimens include Cis plus FU, Doxo plus FU, FU plus $\mathrm{Me}$, and Epi plus FU. A third drug that was added in the triplet regimens was usually Doxo, FU, Me, Eto, or MMC. The median OS in doublet regimen groups ranged from 3.3 months to 8.61 months, while that in triplet groups was between 5.5 months and 8.5 months $[29,30]$. The ORR in doublet regimen groups ranged from 0 to $51 \%$ [30, 40], while that in triplet groups ranged from 12 to $39 \%$ [27, 29]. A serious new generation of chemotherapeutic drugs such as Epi, DTX and PTX, Ox, Iri, Cap, and S-1 were also added into doublet or triplet chemotherapy in treating patients with advanced gastric cancer. Epi, Cap or S-1, and Ox replace Doxo, FU, and Cis in new doublet regimens, respectively. Also, DTX or PTX and Iri were added in novel doublet regimens, respectively. Similarly, a third new chemotherapeutic drug was added into traditional or new doublet regimens, resulting in a series of new triplet chemotherapy regimens. These new triplet regimens were widely compared with traditional or new doublet regimens in various RCTs in advanced gastric cancer. The common triplet regimens include Epi plus Cis plus 5-FU/Cap, DTX/PTX plus Cis/Ox plus 5-FU/Cap/S-1, and Cis plus Iri plus FU. The new doublet regimens have an OS that ranged from 7.1 to 15.3 months and an ORR that ranged from 18.4 to $56 \%[12,24,36]$. The triplet regimens have an OS that ranged from 8.3 to 17.3 months and an ORR that ranged from 27 to $59.3 \%$ [12, $21,24,33]$. Both OS and ORR were significantly improved in new doublet and triplet regimens [2, 42].

There were more than 20 triplet regimens and doublet regimens that were included in this meta-analysis. We divided these chemotherapy regimens into seven kinds, that is, whether two of the chemotherapeutic drugs present in triplet regimens were identical or homogenous to doublet regimens. These regimens included fluorouracil-based, platinum-based, MMC-based, anthracycline-based, taxane-based, and other chemotherapies. Because of the absence of a study that compares irinotecan-based triplet regimens with non-irinotecan-based doublet regimen, we also classified a kind of "irinotecan," that is, irinotecanbased double regimens.

This systematic review and meta-analysis revealed that fluorouracil-based triplet regimens were superior to doublet regimens in terms of OS and ORR but not PFS. These results were consistent with the previous meta-analysis [9].
The pooled result of the improved PFS in fluorouracilbased triplet chemotherapy was not completely convincing due to the following reasons: high heterogeneity and relatively small samples. The HR (0.80) of OS may still be probable and is considered clinically meaningful because of the presence of relatively large samples. Platinum-based triplet regimens improved OS but not PFS and ORR compared with doublet regimen. These results were in line with previous meta-analysis and also were similar with another. However, MMC-based and anthracycline-based triplet regimens improved neither primary nor second outcomes. What should be noticed is that the results of the pooled analysis of anthracycline-based triplet regimens benefiting patients with advanced gastric cancer remain controversial. An early meta-analysis confirmed that anthracycline-based triplet regimens could improve OS [41]. Nevertheless, a recent meta-analysis holds the doubtful conclusion [9]. Moreover, another recent network meta-analysis indicates that anthracycline-based triplet chemotherapy did not improve OS and PFS compared with fluorouracil-based doublet chemotherapy [8]. Though our meta-analysis included RCTs and had no heterogeneity, the overall patient samples were small. Thus, it is still hard to confirm if patients did benefit from anthracycline-based triplet regimens.

In our meta-analysis, taxane-based triplet regimens did not improve OS but improved ORR for patients with advanced gastric cancer. Whether taxane-based triplet regimens improve survival is the mostly disputed topic among previous meta-analysis. A meta-analysis concluded that taxane-based triplet regimens significantly improved OS, PFS, and ORR of patients with advanced gastric cancer [9]. However, a network meta-analysis revealed that taxane-based triplet regimens did not improve OS and PFS compared with fluorouracil-based doublet chemotherapy [8]. The former included one more trial than the latter. Additionally, our metaanalysis also enrolled new large samples of an RCT accounting for $24.9 \%$ of all included trials [12]. The different RCT samples among several meta-analysis contributed the various outcomes. A more recent study with 741 patients failed to prove that taxane-based triplet regimens could improve OS, PFS, and ORR compared with doublet regimens [12]. This study had majority of weight of taxane-based subgroup in our meta-analysis and was related to the negative outcome of OS. Nevertheless, our pooled analysis still demonstrated that taxane-based triplet regimens improved ORR of patients with advanced gastric cancer. Lastly, other drug-based regimens did not improve OS, PFS, and ORR in patients with advanced gastric cancer, and irinotecan-based chemotherapy regimens also did not improve the ORR.

To the best of our knowledge, this meta-analysis firstly and separately analyzed Asian and Western patients, that is, whether they can get more benefit from triplet 
chemotherapy compared with doublet chemotherapy. The pooled result revealed that Western patients' OS improved with triplet chemotherapy while Asian patients' OS did not. There were 11 trials including 1630 patients and 10 trials including 1883 patients in Asia and Western, respectively, in our meta-analysis. Moreover, both subgroups had low heterogeneity $\left(\mathrm{I}^{2}=30 \%\right.$ in Asia and $35 \%$ in Western group). We also individually analyze two trials as a subgroup that included patients both from Asia and Western; however, detailed geographic data of patients were not taken. Therefore, the results of the different improvement of OS between Asian and Western patients could be highly robust. Studies have shown that the proportion of patients with advanced gastric cancer in Asia receiving second-line treatment were higher than that in Western patients [43-48]. Furthermore, a meta-analysis showed that the 1 -year OS rate of advanced gastric cancer will improve by $10 \%$ for every $10 \%$ increase in patients receiving second-line chemotherapy [49]. And the first-line use of triplet chemotherapy may lead to drug resistance to basic chemotherapeutic drugs and reduce the choice of follow-up chemotherapeutic drugs. Hence, it is most likely that further treatment following the first-line treatment in Asia confounded the outcomes of triplet combination chemotherapy.

Subgroup analysis of the same chemotherapy regimens indicated that triplet chemotherapy regimens improve the OS and ORR, while PFS had negative result. The overall PFS analysis showed that triplet chemotherapy regimens could significantly improve PFS, but the subgroup analysis of the same regimens showed the negative result, which may be related to deletion of studies that have only one type of triplet and doublet chemotherapy regimens.

Some limitations of the present analysis should be acknowledged. First is the difference in the parameters of patients, regimens, and dose induced to heterogeneity among some of the included trials. Though we used the random effects model to compute the estimates, the heterogeneity might potentially affect the results. Second, patients receiving second-line treatments were not reported; hence, the possible impact on outcomes could not be considered. However, second-line treatments were not related to the PFS in first-line chemotherapy. Third, our meta-analysis was based on the aggregate data from longitudinal RCTs rather than individual patient data. Therefore, discrimination in individual baseline parameters cannot be regulated. Fourth, some of the included trials in our analysis did not provide the data of OS, PFS, TTF, and toxicity, especially several abstracts from ASCO and ESMO conferences. Insufficient amount of data might potentially influence the analysis.

\section{Conclusion}

In conclusion, compared with doublet chemotherapy, triplet chemotherapy, as a first-line treatment, improved OS, PFS, TTP, and OS in patients with advanced gastric cancer among overall populations, especially for fluoropyrimidine- or platinum-based triplet chemotherapy, which showed a significant improvement in OS. In the subgroup analyses, triplet chemotherapy improved OS in Western but not in Asian patients.

\section{Supplementary information}

Supplementary information accompanies this paper at https://doi.org/10. 1186/s12885-019-6294-9.

Additional file 1: Figure S1. Subgroup analysis of overall survival for triplet chemotherapy versus doublet chemotherapy.

Additional file 2: Figure S2. Subgroup analysis of progression-free survival for triplet chemotherapy versus doublet chemotherapy.

Additional file 3: Figure S3. Subgroup analysis of objective response rate for triplet chemotherapy versus doublet chemotherapy.

Additional file 4: Figure S4. Comparison of the same chemotherapy regimens of overall survival for triplet chemotherapy versus doublet chemotherapy.

Additional file 5: Figure S5. Comparison of the same chemotherapy regimens of progression-free survival for triplet chemotherapy versus doublet chemotherapy.

Additional file 6: Figure S6. Comparison of the same chemotherapy regimens of objective response rate for triplet chemotherapy versus doublet chemotherapy.

\section{Abbreviations}

5-FU: 5-fluorouracil; AEs: Adverse events; ASCO: American Society of Clinical Oncology; Cap: Capecitabine; CENTRAL: Cochrane Register of Controlled Trials; Cis: Cisplatin; D: Doxorubicin; DCF: Docetaxel and cisplatin plus fluorouracil; E: Epirubicin; E: Etoposide; ECOG: Eastern Cooperative Oncology Group; ESMO: European Society for Medical Oncology; F: Fluoropyrimidine; HER2: Human epidermal growth factor receptor 2; I: Irinotecan; ITT: Intentionto-treat; Me: Semustine; MMC: Mitomycin; Mtx: Methotrexate; ORR: Objective response rate; Ox: Oxaliplatin; PFS: Progression-free survival; PRISMA: Preferred Reporting Items for Systematic Reviews and MetaAnalyses; PS: Performance status; RCTs: Randomized controlled trials; SE: Standard error; T: Taxane; Te: Tegafur; TTP: Time to progress; U: Uracil

\section{Acknowledgements}

Not applicable.

\section{Authors' contributions}

$X G, F Z$ and $X M$ contributed equally to this work. $X G$ and $F Z$ analysed and interpreted data, drafted the manuscript. XM made acquisition of data, performed statistical analysis. GS, DR, FZ, FD, ZW, RA and XY participated in studies selection and data extraction and provided statistical expertise. JunhuiZ and JZhao conceived of the study, participated in its design, analysed and interpreted the data. All authors read and approved the final manuscript.

\section{Funding}

This work was supported by grants from the Thousand Talents of Program of High-end Innovation of Qinghai Province in China (for Dr. Jiuda Zhao) and the Qinghai province Medical Gene Detection Technology Platform (2018-ZJT02). The sponsors played no role in the study design, data collection, analysis, or decision to submit the article for publication.

Availability of data and materials Not applicable. 


\section{Ethics approval and consent to participate}

Not applicable.

\section{Consent for publication}

Not applicable.

\section{Competing interests}

The authors declare that they have no competing interests.

\section{Author details}

${ }^{1}$ Affiliated Hospital of Qinghai University, Affiliated Cancer Hospital of Qinghai University, Xining 810000, China. ${ }^{2}$ Shouguang Hospital of Traditional Chinese Medicine, Weifang 262700, China. ${ }^{3}$ Department of Medical Oncology, Cancer hospital, Chinese academy of medical sciences, Peking Union Medical College, Beijing 100021, China. ${ }^{4}$ Peking University Cancer Hospital and Institute, Beijing 100142, China.

\section{Received: 12 February 2019 Accepted: 25 October 2019} Published online: 20 November 2019

\section{References}

1. Bray F, Ferlay J, Soerjomataram I, Siegel RL, Torre LA, Jemal A. Global cancer statistics 2018: GLOBOCAN estimates of incidence and mortality worldwide for 36 cancers in 185 countries. CA Cancer J Clin. 2018;68:394-424.

2. Wagner AD, Syn NL, Moehler M, Grothe W, Yong WP, Tai BC, et al. Chemotherapy for advanced gastric cancer. Cochrane Database Syst Rev. 2017:8:CD004064.

3. Van Cutsem E, Sagaert X, Topal B, Haustermans K, Prenen H, Van Cutsem E, Sagaert X, Topal B, et al. Gastric cancer. Lancet. 2016;388(10060):2654-64

4. Ajani JA, Farjah F, Johung KJ, Paluri RK, D'Amico TA, Gerde H, Keswani RN. National Comprehensive Cancer Network (NCCN) Clinical Practice Guidelines in Oncology, Gastric Cancer. 2018 version 2. Available from: https://www.nccn.org/professionals/physician_gls/default.aspx\#gastric.

5. Japanese Gastric Cancer Association. Japanese gastric cancer treatment guidelines 2014 (ver. 4). Gastric Cancer. 2017;20(1):1-19.

6. Wadhwa R, Song S, Lee JS, Yao Y, Wei Q, Ajani JA. Gastric cancer-molecular and clinical dimensions. Nat Rev Clin Oncol. 2013;10(11):643-55.

7. Bang YJ, Van Cutsem E, Feyereislova A, Chung HC, Shen L, Sawaki A, et al. Trastuzumab in combination with chemotherapy versus chemotherapy alone for treatment of HER2-positive advanced gastric or gastrooesophageal junction cancer (ToGA): a phase 3, open-label, randomised controlled trial. Lancet. 2010;376(9742):687-97.

8. Ter VE, Haj MN, van Valkenhoef G, Ngai LL, RMA M, Anderegg MC, et al. The Efficacy and Safety of First-line Chemotherapy in Advanced Esophagogastric Cancer: A Network Meta-analysis. J Natl Cancer Inst. 2016;108(10).

9. Mohammad NH, ter VE, Ngai L, Mali R, van Oijen MG, van Laarhoven HW. Optimal first-line chemotherapeutic treatment in patients with locally advanced or metastatic esophagogastric carcinoma: triplet versus doublet chemotherapy: a systematic literature review and meta-analysis. Cancer Metastasis Rev. 2015;34(3):429-41.

10. Smyth EC, Verheij M, Allum W, Cunningham D, Cervantes A, Arnold D. Gastric cancer: ESMO Clinical Practice Guidelines for diagnosis, treatment and follow-up. Ann Oncol. 2016;27(suppl 5):v38-38v49.

11. Wang F H, Shen L, Li J, et al. The Chinese Society of Clinical Oncology (CSCO): clinical guidelines for the diagnosis and treatment of gastric cancer. Cancer Communications. 2019;39(1):10.

12. Yamada Y, Boku N, Mizusawa J, Iwasa S, Kadowaki S, Nakayama N, et al. Phase III study comparing triplet chemotherapy with S-1 and cisplatin plus docetaxel versus doublet chemotherapy with $\mathrm{S}-1$ and cisplatin for advanced gastric cancer (JCOG1013); 2018. p. 4009-9.

13. Laterza MM, Pompella L, Petrillo A, Tirino G, Pappalardo A, Orditura M, et al. Efficacy of a triplet and doublet-based chemotherapy as first-line therapy in patients with HER2-negative metastatic gastric cancer: a retrospective analysis from the clinical practice. Med Oncol. 2017;34(11):186.

14. Wang J, Xu R, Li J, Bai Y, Liu T, Jiao S, et al. Randomized multicenter phase III study of a modified docetaxel and cisplatin plus fluorouracil regimen compared with cisplatin and fluorouracil as first-line therapy for advanced or locally recurrent gastric cancer. Gastric Cancer. 2016;19(1):234-44.

15. Liberati A, Altman DG, Tetzlaff J, Mulrow C, Gøtzsche PC, loannidis JP, et al. The PRISMA statement for reporting systematic reviews and meta-analyses of studies that evaluate healthcare interventions: explanation and elaboration. BMJ. 2009;339:b2700

16. Page MJ, Moher D. Evaluations of the uptake and impact of the preferred reporting items for systematic reviews and meta-analyses (PRISMA) statement and extensions: a scoping review. Syst Rev. 2017;6(1):263.

17. Moher D, Liberati A, Tetzlaff J, Altman DG. Preferred reporting items for systematic reviews and meta-analyses: the PRISMA statement. BMJ. 2009;339:b2535.

18. Sterne JA, Egger M. Funnel plots for detecting bias in metaanalysis: guidelines on choice of axis. J Clin Epidemiol. 2011;54(10):1046-55.

19. Higgins JP, Altman DG, Gøtzsche PC, Jüni P, Moher D, Oxman AD, et al. The Cochrane Collaboration's tool for assessing risk of bias in randomised trials. BMJ. 2011;343:d5928.

20. Ajani JA, Fodor MB, Tjulandin SA, Moiseyenko VM, Chao Y, Cabral FS, et al. Phase II multi-institutional randomized trial of docetaxel plus cisplatin with or without fluorouracil in patients with untreated, advanced gastric, or gastroesophageal adenocarcinoma. J Clin Oncol. 2005;23(24):5660-7.

21. Al-Batran SE, Pauligk C, Homann N, Hartmann JT, Moehler M, Probst S, et al. The feasibility of triple-drug chemotherapy combination in older adult patients with oesophagogastric cancer: a randomised trial of the Arbeitsgemeinschaft Internistische Onkologie (FLOT65+). Eur J Cancer. 2013;49(4):835-42.

22. Guimbaud R, Louvet C, Ries P, Ychou M, Maillard E, André T, et al. Prospective, randomized, multicenter, phase III study of fluorouracil, leucovorin, and irinotecan versus epirubicin, cisplatin, and capecitabine in advanced gastric adenocarcinoma: a French intergroup (Fédération Francophone de Cancérologie Digestive, Fédération Nationale des Centres de Lutte Contre le Cancer, and Groupe Coopérateur Multidisciplinaire en Oncologie) study. J Clin Oncol. 2014;32(31):3520-6.

23. Park SH, Nam E, Park J, Cho EK, Shin DB, Lee JH, et al. Randomized phase II study of irinotecan, leucovorin and 5-fluorouracil (ILF) versus cisplatin plus ILF (PILF) combination chemotherapy for advanced gastric cancer. Ann Oncol. 2008;19(4):729-33.

24. Roth AD, Fazio N, Stupp R, Falk S, Bernhard J, Saletti P, et al. Docetaxel, cisplatin, and fluorouracil; docetaxel and cisplatin; and epirubicin, cisplatin, and fluorouracil as systemic treatment for advanced gastric carcinoma: a randomized phase II trial of the Swiss group for clinical Cancer research. J Clin Oncol. 2007:25(22):3217-23.

25. Van Cutsem E, Boni C, Tabernero J, Massuti B, Middleton G, Dane F, et al. Docetaxel plus oxaliplatin with or without fluorouracil or capecitabine in metastatic or locally recurrent gastric cancer: a randomized phase II study. Ann Oncol. 2015;26(1):149-56.

26. Van Cutsem E, Moiseyenko VM, Tjulandin S, Majlis A, Constenla M, Boni C, et al. Phase III study of docetaxel and cisplatin plus fluorouracil compared with cisplatin and fluorouracil as first-line therapy for advanced gastric cancer: a report of the V325 study group. J Clin Oncol. 2006;24(31):4991-7.

27. Vanhoefer U, Rougier P, Wilke H, Ducreux MP, Lacave AJ, Van Cutsem E, et al. Final results of a randomized phase III trial of sequential high-dose methotrexate, fluorouracil, and doxorubicin versus etoposide, leucovorin, and fluorouracil versus infusional fluorouracil and cisplatin in advanced gastric cancer: a trial of the European Organization for Research and Treatment of Cancer gastrointestinal tract Cancer cooperative group. J Clin Oncol. 2000;18(14):2648-57.

28. Cullinan SA, Moertel CG, Fleming TR, Rubin JR, Krook JE, Everson LK, et al. A comparison of three chemotherapeutic regimens in the treatment of advanced pancreatic and gastric carcinoma. Fluorouracil vs fluorouracil and doxorubicin vs fluorouracil, doxorubicin, and mitomycin. JAMA. 1985;253(14):2061-7.

29. Douglass HO, Lavin PT, Goudsmit A, Klaassen DJ, Paul AR. An eastern cooperative oncology group evaluation of combinations of methyl-CCNU, mitomycin C, Adriamycin, and 5-fluorouracil in advanced measurable gastric cancer (EST 2277). J Clin Oncol. 1984;2(12):1372-81.

30. Kim NK, Park YS, Heo DS, Suh C, Kim SY, Park KC, et al. A phase III randomized study of 5-fluorouracil and cisplatin versus 5-fluorouracil, doxorubicin, and mitomycin $\mathrm{C}$ versus 5 -fluorouracil alone in the treatment of advanced gastric cancer. Cancer. 1993;71(12):3813-8.

31. Kim T, Choi SJ, e.a. Ahn JH. A prospective randomized phase III trial of 5fluorouracil and cisplatin (FP) versus epirubicin, cisplatin, and 5FU (ECF) in the treatment of patients with previously untreated advanced gastric cancer (AGC). Eur J Cancer. 2001;37(Suppl 6):S314.

32. Koizumi W, Fukuyama Y, Fukuda T, Akiya T, Hasegawa K, Kojima $Y$, et al. Randomized phase II study comparing mitomycin, cisplatin plus doxifluridine with cisplatin plus doxifluridine in advanced unresectable gastric cancer. Anticancer Res. 2004;24(4):2465-70. 
33. A randomized, comparative study of combination chemotherapies in advanced gastric cancer: 5-fluorouracil and cisplatin (FP) versus 5fluorouracil, cisplatin, and 4'-epirubicin (FPEPIR). Kyoto Research Group for Chemotherapy of Gastric Cancer (KRGCGC). Anticancer Res. 1992; 12(6B):1983-8

34. Li XD, Shen H, Jiang JT, Zhang HZ, Zheng X, Shu YQ, et al. Paclitaxel based vs oxaliplatin based regimens for advanced gastric cancer. World J Gastroenterol. 2011;17(8):1082-7.

35. Lin R, et al. Phase Illb trial of fluorouracil, leucovorin,oxaliplatin, and paclitaxel (POF) compared with fluorouracil, leucovorin, and irinotecan (IF) as first-line treatment for advanced gastric cancer (AGC). J Clin Oncol. 2009;27(suppl; abstr e 15642).

36. Roth A, Kolaric K, Zupanc D, Oresic V, Roth A, Ebling Z. High doses of 5fluorouracil and epirubicin with or without cisplatin in advanced gastric cancer: a randomized study. Tumori. 1999;85(4):234-8.

37. Thuss-Patience PC, Kretzschmar A, Repp M, Kingreen D, Hennesser D, Micheel $S$, et al. Docetaxel and continuous-infusion fluorouracil versus epirubicin, cisplatin, and fluorouracil for advanced gastric adenocarcinoma: a randomized phase II study. J Clin Oncol. 2005;23(3):494-501.

38. Maiello $E$, et al. Epirubicin (E) in combination with cisplatin (CDDP) and capecitabine (C) versus docetaxel (D) combined with 5-fluorouracil (\%-FU) by continuous infusion as front-line therapy in patients with advanced gastric cancer (AGC): preliminary results of a randomized. 2011;29(4 suppl; abstr 97).

39. Yun J, Lee J, Park SH, Park JO, Park YS, Lim HY, et al. A randomised phase II study of combination chemotherapy with epirubicin, cisplatin and capecitabine (ECX) or cisplatin and capecitabine (CX) in advanced gastric cancer. Eur J Cancer. 2010;46(5):885-91.

40. Kikuchi K, Wakui A, Shimizu H, Kunii Y. Randomized controlled study on chemotherapy with 5-FD, ADM plus CDDP in advanced gastric carcinoma. Gan To Kagaku Ryoho. 1990;17(4 Pt 1):655-62.

41. Wagner AD, Grothe W, Haerting J, Kleber G, Grothey A, Fleig WE. Chemotherapy in advanced gastric cancer: a systematic review and metaanalysis based on aggregate data. J Clin Oncol. 2006;24(18):2903-9.

42. Shen L, Shan YS, Hu HM, Price TJ, Sirohi B, Yeh KH, et al. Management of gastric cancer in Asia: resource-stratified guidelines. Lancet Oncol. 2013:14(12):e535-47.

43. Cunningham D, Starling N, Rao S, Iveson T, Nicolson M, Coxon F, et al. Capecitabine and oxaliplatin for advanced esophagogastric cancer. N Engl J Med. 2008;358(1):36-46.

44. Boku N, Yamamoto S, Fukuda H, Shirao K, Doi T, Sawaki A, et al. Fluorouracil versus combination of irinotecan plus cisplatin versus S-1 in metastatic gastric cancer: a randomised phase 3 study. Lancet Oncol. 2009:10(11):1063-9.

45. Koizumi W, Narahara H, Hara T, Takagane A, Akiya T, Takagi M, et al. S-1 plus cisplatin versus S-1 alone for first-line treatment of advanced gastric cancer (SPIRITS trial): a phase III trial. Lancet Oncol. 2008;9(3):215-21.

46. Ajani JA, Rodriguez W, Bodoky G, Moiseyenko V, Lichinitser M, Gorbunova V, et al. Multicenter phase III comparison of cisplatin/S-1 with cisplatin/ infusional fluorouracil in advanced gastric or gastroesophageal adenocarcinoma study: the FLAGS trial. J Clin Oncol. 2010;28(9):1547-53.

47. Van Cutsem E, de Haas S, Kang YK, Ohtsu A, Tebbutt NC, Ming XJ, et al. Bevacizumab in combination with chemotherapy as first-line therapy in advanced gastric cancer: a biomarker evaluation from the AVAGAST randomized phase III trial. J Clin Oncol. 2012;30(17):2119-27.

48. Kim R, Tan A, Choi M, El-Rayes BF. Geographic differences in approach to advanced gastric cancer: is there a standard approach. Crit Rev Oncol Hematol. 2013;88(2):416-26.

49. Hsu C, Shen YC, Cheng CC, Cheng AL, Hu FC, Yeh KH. Geographic difference in safety and efficacy of systemicchemotherapy for advanced gastric or gastroesophagealcarcinoma: a meta-analysis and meta-regression. Gastric Cancer. 2012;15(3):265-80.

\section{Publisher's Note}

Springer Nature remains neutral with regard to jurisdictional claims in published maps and institutional affiliations.

\section{Ready to submit your research? Choose BMC and benefit from:}

- fast, convenient online submission

- thorough peer review by experienced researchers in your field

- rapid publication on acceptance

- support for research data, including large and complex data types

- gold Open Access which fosters wider collaboration and increased citations

- maximum visibility for your research: over $100 \mathrm{M}$ website views per year

At BMC, research is always in progress.

Learn more biomedcentral.com/submissions 